Artículos científicos

\title{
Análisis descriptivo de las diferencias por sexo en los estudiantes de licenciatura de la Universidad de Guadalajara
}

\section{A Descriptive Analysis of the Differences by Sex in Undergraduate Students at the University of Guadalajara}

Análise descritiva das diferenças por sexo em alunos de graduação da Universidade de Guadalajara

Salvador Sandoval Bravo

Universidad de Guadalajara, Departamento de Métodos Cuantitativos, México salvador.sandoval@academicos.udg.mx https://orcid.org/0000-0001-9434-6536

Pedro Luis Celso Arellano Universidad de Guadalajara, Departamento de Métodos Cuantitativos, México pedro.celso@academicos.udg.mx https://orcid.org/0000-0003-3856-6344

Victor Hugo Gualajara Estrada

Universidad de Guadalajara, Departamento de Métodos Cuantitativos, México victor.gualajara@academicos.udg.mx https://orcid.org/0000-0002-5405-3779 


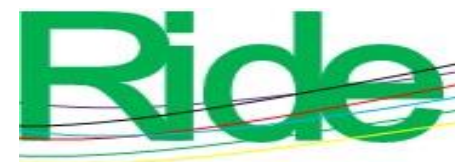

Revista Iberoamericana para la Investigación y el Desarrollo Educativo ISSN $2007-7467$

\section{Resumen}

Existen características biológicas y psicológicas que distinguen a hombres y mujeres. Si bien tales diferencias se manifiestan en todas las áreas de la vida y en todas sus etapas, particularmente se evidencia en la etapa universitaria, donde se suscitan en los jóvenes cambios personales trascendentales. El presente estudio tiene como finalidad hacer un estudio comparativo para identificar y cuantificar las diferencias por sexo que existen entre los estudiantes de licenciatura de la Universidad de Guadalajara. Se analizaron distintas variables de diversa índole: desde demográficas y académicas hasta de orientación sexual, adicciones y de bienestar general. Los datos se obtuvieron a partir de una consulta online e incluye una muestra de 5245 alumnos de licenciatura de los diferentes centros universitarios de la zona metropolitana de Guadalajara y regionales de Jalisco. Se realizó un análisis gráfico, descriptivo e inferencial de las diferencias por sexo. Los resultados revelan diferencias significativas en la mayoría de los rubros analizados; por ejemplo, la proporción de mujeres que son madres es mayor que la de hombres que son padres (4.57 \% vs. $2.69 \%$ ); las mujeres tienen una calificación promedio mayor a la de los hombres (86.40 vs. 84.75), y el porcentaje de quienes han probado alguna droga es mayor en hombres que en mujeres ( $34.67 \%$ vs. $22.57 \%)$.

Palabras clave: adicción, bienestar del estudiante, estudiantes universitarios, rendimiento escolar, sexo.

\section{Abstract}

There are biological and psychological characteristics that distinguish men and women. Although such differences are manifested in all areas of life and in all its stages, it is particularly evident in the university stage, where transcendental personal changes occur in young people. The present study aims to carry out a comparative study to identify and quantify the differences by sex that exist among undergraduate students at the University of Guadalajara. Different variables of various kinds were analyzed, from demographic and academic to sexual orientation, addictions and general well-being. The data was obtained from an online consultation and includes a sample of 5245 undergraduate students from different university centers in the metropolitan area of Guadalajara and regional centers in the state of Jalisco. A graphic, descriptive and inferential analysis of the differences by sex 


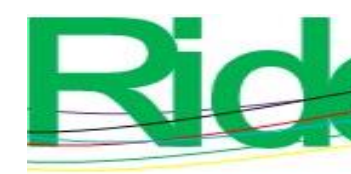

Revista Iberoamericana para la
Investigación y el Desarrollo Educativo
ISSN $2007-7467$

was carried out. The results reveal significant differences in most of the items analyzed; for example, the proportion of women who are mothers is higher than that of men who are fathers (4.57\% vs. $2.69 \%)$; women have a higher average score than men (86.40 vs. 84.75$)$, and the percentage of individuals who have tried a drug is higher in men than in women $(34.67 \%$ vs. $22.57 \%$ ), among other findings.

Keywords: addiction, student welfare, college students, academic performance, sex.

\section{Resumo}

Existem características biológicas e psicológicas que distinguem homens e mulheres. Embora tais diferenças se manifestem em todas as áreas da vida e em todas as suas etapas, é particularmente evidente na fase universitária, onde ocorrem mudanças pessoais transcendentais nos jovens. O objetivo deste estudo é realizar um estudo comparativo para identificar e quantificar as diferenças por sexo que existem entre os alunos de graduação da Universidade de Guadalajara. Diferentes variáveis de vários tipos foram analisadas: desde demográficas e acadêmicas até orientação sexual, vícios e bem-estar geral. Os dados foram obtidos a partir de uma consulta online e incluem uma amostra de 5.245 alunos de graduação de diferentes centros universitários da área metropolitana de Guadalajara e áreas regionais de Jalisco. Foi realizada uma análise gráfica, descritiva e inferencial das diferenças por sexo. Os resultados revelam diferenças significativas na maioria dos itens analisados; por exemplo, a proporção de mulheres que são mães é maior do que a de homens que são pais (4,57\% vs. 2,69\%); as mulheres têm pontuação média superior à dos homens (86,40 vs. 84,75), e o percentual de pessoas que já experimentaram alguma droga é maior nos homens do que nas mulheres $(34,67 \%$ vs. $22,57 \%)$.

Palavras-chave: dependência, bem-estar do aluno, estudantes universitários, desempenho escolar, sexo.

Fecha Recepción: Noviembre 2020

Fecha Aceptación: Abril 2021 


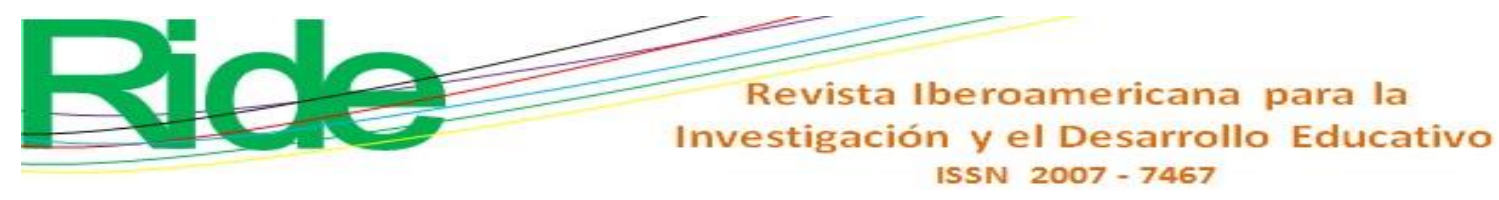

\section{Introducción}

Anteriormente, la educación universitaria era un espacio predominantemente masculino. Los hombres se preparaban en los distintos ámbitos del saber para ocupar los puestos que demandaba el mercado laboral, lograr el éxito profesional y el reconocimiento social; mientras que las mujeres eran relegadas a roles más domésticos como la procreación, atender los deberes del hogar y el cuidado de los hijos. Por fortuna, durante las últimas décadas, la situación ha cambiado y se ha logrado un viraje que ha favorecido el ingreso cada vez mayor de mujeres a los distintos niveles educativos, sobre todo en la educación superior, donde es cada vez mayor el número y porcentaje de mujeres que acceden y egresan de las universidades, gracias a lo cual se han podido incorporar exitosamente a la esfera profesional (Papadópulos y Radakovich, 2006). Cabe destacar que, en el rubro académico, cada vez se fortalecen más las estructuras de equilibrio entre sexos, que permiten la convivencia y competencia igualitaria entre hombres y mujeres.

De esta manera, la participación cada vez mayor de las mujeres en la educación superior ha contribuido a revertir las condiciones de sumisión y discriminación en que se encontraban, y ha causado que las posibilidades de integrarse con todo su potencial en el mercado laboral, lograr independencia económica y fortalecer su desarrollo personal y profesional sean más altas (Aponte, 2008). En el futuro, este proceso se traducirá en la construcción de estructuras sociales sólidas con mayor paridad entre los sexos en todas las áreas de la actividad humana, donde mujeres y hombres trabajen armoniosamente y en condiciones igualitarias en aras del bienestar común.

Ahora bien, aunque recientemente se ha acortado la brecha entre sexos a nivel universitario, todavía subsisten diferencias notorias debido a consideraciones de tipo biológico, psicológico o cultural que inciden de diversas formas (por ejemplo, la asignación de roles familiares). Y lo mismo sucede en el mercado laboral, donde todavía perviven evidentes contrastes ya sea en el tipo de empleo o en las condiciones laborales, ya sea en el acceso a puestos de decisión y poder o en el salario (García, 2005).

Uno de los aspectos más sobresalientes en cuanto al equilibrio entre sexos es el aumento sostenido de mujeres en la matrícula, permanencia y egreso de las universidades. De hecho, en la mayoría de los países latinoamericanos, la población femenina ya rebasa a la población masculina, en algunos casos en proporciones significativas (Papadópulos y Radakovich, 2006). En México, existe un cierto equilibrio porcentual a nivel universitario, 


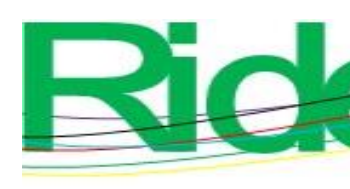

Revista Iberoamericana para la Investigación y el Desarrollo Educativo ISSN $2007-7467$

aunque es ligeramente mayor la población femenina (Barrón, Madera y Cayeros, 2018). En cuanto a la distribución de la matrícula por área del saber, también persisten algunas diferencias significativas. Por ejemplo, en México hay bastiones masculinos como el área de ingeniería, pero también hay reductos femeninos como enfermería, trabajo social y turismo; no obstante, también existen áreas donde no existen diferencias considerables entre ambos sexos como en muchas licenciaturas de ciencias de la salud y ciencias económicoadministrativas (Huerta, 2017).

Las diferencias entre sexos en el rendimiento escolar a nivel licenciatura han sido analizadas por diversas investigaciones con distintos enfoques; los resultados muchas veces son contradictorios. Algunos estudios se focalizan en medir habilidades cognitivas, otros le dan preponderancia a la inteligencia emocional, algunos más al razonamiento espacial y abstracto, un grueso de estudios se enfoca en las habilidades verbales, otro tanto en la aplicación efectiva de técnicas de estudio... Y si bien es cierto que es posible encontrar diferencias significativas, también pueden identificarse similitudes importantes (Pantoja y Alcaide, 2013). Tampoco en cuanto a las notas finales por materias, o en los porcentajes de ingreso, permanencia o egreso de las carreras, hay resultados concluyentes y estos pueden variar de un estudio a otro (Oyarzún, Estrada, Pino y Oyarzún, 2012). Huelga decir que existen otras variables que inciden, en mayor o menor grado, en el rendimiento académico, tales como la motivación, las habilidades sociales, el autocontrol, los estilos de personalidad, y otros factores sociales y culturales que pueden variar o no dependiendo del sexo de los estudiantes (Torres, 2011).

Por otra parte, en el ambiente universitario suelen afianzarse, tanto en hombres como en mujeres, comportamientos sexuales como la orientación sexual de los estudiantes (heterosexuales, homosexuales y bisexuales, principalmente) que se manifiestan desde la niñez y la adolescencia, y que permean la personalidad y el desarrollo de los jóvenes en todos los ámbitos de la vida, al igual que condicionan su salud física y emocional (Barra, 2013; Oswalt y Wyatt, 2013; Paredes y Polanski, 2016). Por tal motivo, es importante reconocer que existe una pluralidad manifiesta de orientaciones sexuales en la esfera universitaria, y que en años recientes existe aceptación, tolerancia y respeto a dichas diferencias.

En otro orden de ideas, todos los universitarios, ya sean hombres o mujeres, están sujetos a presiones sociales y económicas, estrés emocional, tensiones académicas y problemas familiares; estos factores pueden provocar o acentuar el consumo de drogas, 


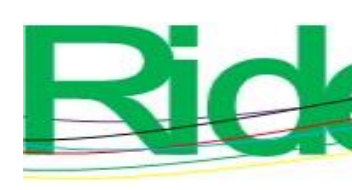

Revista Iberoamericana para la Investigación y el Desarrollo Educativo ISSN 2007 - 7467

tabaco o alcohol en los estudiantes (Gómez, Landeros, Noa y Martínez, 2017). Sin duda la incidencia creciente en las adicciones en el entorno académico es una preocupación permanente y un problema de salud que puede tener consecuencias importantes: reducir el rendimiento escolar de los jóvenes, ocasionar deserción escolar o incluso causar daños irreversibles en la salud de los jóvenes. Es importante conocer la situación a detalle e implementar políticas educativas que permitan controlar y reducir las adicciones a nivel licenciatura (Fernández et al., 2013; Motos, Cortés, Giménez y Cadaveira, 2015).

Otra cuestión de interés en lo que respecta al sexo de los estudiantes de licenciatura es analizar el bienestar desde una perspectiva psicológica y social que considere la relación integral de los individuos con su entorno familiar, social y laboral (Barrantes y Ureña, 2015; Del Valle, Hormaechea y Urquijo, 2015). Dados los roles tradicionalmente asignados a hombres y mujeres pueden distinguirse niveles diferentes o similares en algunos indicadores de bienestar, como en la autoaceptación, las relaciones positivas con otras personas, la autonomía, el propósito de vida, el crecimiento personal, entre otros. Los indicadores anteriores pueden reflejarse en la percepción personal de los universitarios respecto a su felicidad; y su nivel de satisfacción personal con respecto a su propia salud, su economía, su ocupación, sus relaciones familiares, sus relaciones amistosas, su tiempo libre, el ambiente escolar, etcétera (Del Valle et al., 2015; Lara, Saldaña, Fernández y Delgadillo, 2015).

Así pues, considerando los aspectos referenciados brevemente en los párrafos anteriores, el presente estudio tiene como objetivo realizar un análisis comparativo a nivel descriptivo de los contrastes y similitudes por sexo que hay entre los alumnos de licenciatura de la Universidad de Guadalajara (UdeG). Cabe recalcar que esta investigación se focaliza en las diferencias por sexo, no por género. La primera alude a las diferencias y características biológicas, anatómicas, fisiológicas y genéticas de los seres humanos que definen a los hombres y a las mujeres, mientras que la segunda se refiere a la serie de ideas, conductas y atributos que una sociedad dada considera apropiados para cada sexo (Abad, Ramírez, Fernandes y Ramirez, 2019). En este sentido, cada una de las variables analizadas en este trabajo hacen una clara distinción entre hombres y mujeres sin mencionar este conjunto de concepciones, comportamientos y atribuciones social y culturalmente asociadas al género.

Se examinaron diversos indicadores que incluyen variables de tipo demográfica, académica, de orientación sexual, relativas a adicciones y de bienestar general. De los datos que se presentarán se podrá observar que existen variables que no muestran diferencias 


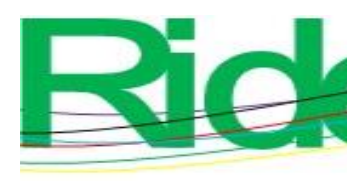

Revista Iberoamericana para la Investigación y el Desarrollo Educativo

ISSN $2007-7467$

significativas por sexo, mientras que en algunas otras las diferencias son muy patentes. La información recabada se obtuvo de una muestra representativa de estudiantes de licenciaturas de los distintos centros universitarios que conforman la UdeG.

Aunado a todo lo anterior, se traza una distinción entre centros universitarios de la zona metropolitana de Guadalajara (ZMG) y los centros universitarios regionales. Como su nombre lo indica, los primeros están situados en la ciudad de Guadalajara, cuya zona metropolitana alberga alrededor de cinco millones de personas; los demás centros universitarios, los centros universitarios regionales, están localizados en ciudades pequeñas, por lo que su población estudiantil está conformada de un porcentaje significativo de poblaciones rurales. En este sentido, existen diferencias importantes entre ambos grupos de estudiantes determinadas por cuestiones familiares, de tradición, de ingreso, de infraestructura urbana, de tamaño de las instalaciones escolares, de acceso a la tecnología y conectividad, de movilidad, entre otros (Parker y Pederzini, 2000; Ramos, Duque y Nieto, 2012); características que, de alguna manera u otra, inciden también en la cuestión de las diferencias entre el sexo de los universitarios, como se podrá observar en los resultados de la presente investigación.

Este trabajo es importante ya que pone de manifiesto el estado actual de las diferencias entre sexos que existen en la población estudiantil de la UdeG a través de un análisis gráfico, descriptivo e inferencial que incluye muchas variables de interés de distintos ámbitos.

\section{Metodología}

La base de datos consultada para realizar este documento se obtuvo a partir de una consulta dirigida a los estudiantes activos de preparatoria y de licenciatura de la UdeG durante el primer semestre del 2019, llevada a cabo por la Federación de Estudiantes Universitarios (FEU), que es la única organización estudiantil reconocida por la UdeG. La consulta recabó información general de los estudiantes, datos demográficos, de salud mental y física, hábitos alimenticios, adicciones, práctica deportiva y discriminación, entre otros.

La consulta se aplicó online a través de la plataforma de formularios mixtos Google Forms, de forma aleatoria, anónima y voluntaria durante el primer semestre de 2019. Se aplicó un cuestionario estructurado que contenía preguntas con respuestas posibles en escala Likert o con respuestas binarias. El cuestionario fue aplicado a todos los estudiantes de 


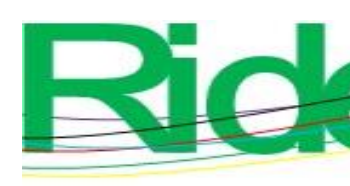

Revista Iberoamericana para la Investigación y el Desarrollo Educativo ISSN $2007-7467$

ciertos grupos al azar de cada centro universitario de la ZMG y regional: aproximadamente $10 \%$ de la población estudiantil de cada centro, por lo que los estudiantes encuestados son de diferentes semestres, turnos, sexos y carreras. A nivel red universitaria, la muestra original consistió en 9638 estudiantes. Para propósitos de este trabajo, esta muestra preliminar se depuró para eliminar cuestionarios mal llenados, con respuestas incompletas, nulas o inconsistentes. De esta forma, la muestra final constó de 5245 elementos de una población de 114314 de alumnos de licenciatura (UdeG, 2019), lo cual garantiza un nivel de confianza de al menos $95 \%$ para las pruebas de estadística inferencial. Se encuestaron al menos 196 estudiantes de cada centro universitario tanto de la ZMG como de los centros universitarios regionales. Los seis centros universitarios de la ZMG son: Centro Universitario de Ciencias Económico Administrativas (Cucea), Centro Universitario de Ciencias Exactas e Ingeniería (Cucei), Centro Universitario de Ciencias Biológicas y Agropecuarias (Cucba), Centro Universitario de Arte, Arquitectura y Diseño (Cuaad), Centro Universitario de Ciencias de la Salud (CUCS) y Centro Universitario de Ciencias Sociales y Humanidades (Cucsh). Los nueve regionales son: Centro Universitario de la Costa (CUCosta), Centro Universitario de la Costa Sur (CUCSur), Centro Universitario de los Altos (CUAltos), Centro Universitario de los Lagos (CULagoS), Centro Universitario de los Valles (CUValles), Centro Universitario de la Ciénega (Cuci), Centro Universitario del Sur (Cusur), Centro Universitario del Norte (CUNorte) y el Centro Universitario de Tonalá (CUTonalá).

Asimismo, se llevaron a cabo 19 pruebas de estadística inferencial, la mayoría de porciones y otras más de medias aritméticas para probar hipótesis referentes a diferencias entre sexos, involucrando una serie de variables de interés: desde indicadores demográficos hasta variables académicas, de preferencias sexuales, de adicción y de bienestar. Estas pruebas contrastan una serie de hipótesis que muestran si existen o no diferencias significativas por sexo teniendo en cuenta una serie de variables como el estado civil, el tener hijos o no, el ingreso promedio semanal, el rendimiento académico promedio, el turno elegido por los estudiantes, el tiempo de permanencia en la escuela, el consumo de drogas, el consumo de alcohol, el consumo de tabaco, el nivel de felicidad, la satisfacción de la salud personal, la satisfacción de la economía personal, la satisfacción ocupacional, la satisfacción respecto a las relaciones familiares, la satisfacción de las relaciones de amistad, la satisfacción respecto al tiempo libre y la satisfacción en relación al ambiente escolar. 


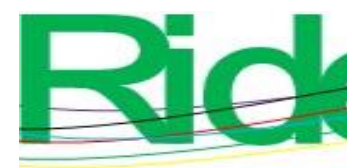

Revista Iberoamericana para la Investigación y el Desarrollo Educativo

ISSN $2007-7467$

Consecuentemente, dada la gran cantidad de variables consideradas y a la heterogeneidad de estas, este trabajo es de carácter general y exploratorio.

También, en la mayoría de las pruebas, se hicieron tres subpruebas: una considerando solo los centros de la ZMG, otra para los centros regionales y otra considerando todos los centros de la red universitaria. El nivel de significancia de todas las pruebas estadísticas fue de $5 \%$. Para hacer estas pruebas se utilizó el software estadístico SPSS. Por supuesto, se construyeron los gráficos descriptivos de todas las pruebas para ilustrar los resultados de la manera más fácil y asequible posible.

Finalmente, cabe señalar que se utilizó el informe de matrícula del primer semestre del año 2019 (UdeG, 2019), que contiene información de los alumnos inscritos en todos los planteles de dicha universidad, tanto del nivel superior como del nivel medio superior.

\section{Resultados y discusión}

A continuación se presenta el análisis descriptivo de los datos recabados por categoría de variables. Los datos van acompañados de algunos comentarios, posibles razones a los resultados mostrados, pero sin pretender demostrar tales especulaciones, simplemente alentar la discusión dada la naturaleza general y exploratoria del estudio.

\section{Indicadores demográficos}

Según la UdeG (2019), en licenciatura $47.09 \%$ de los estudiantes son hombres y $52.91 \%$ son mujeres. La figura 1 muestra la distribución porcentual por sexo en los centros de la red universitaria. Se puede notar que hay centros universitarios con un porcentaje considerablemente mayor de mujeres: CUCS (65.48\%), CUAltos (62.72\%) y CUSur $(60.41 \%)$; mientras que los centros universitarios que cuentan con mayor porcentaje de hombres son Cucei (71.27 \%), CUCSur (49.95\%) y Cucba (48.94 \%). Esta distribución de la matrícula por sexo, en donde el número de mujeres excede al número de hombres, está en concordancia con el comportamiento y tendencia a nivel nacional y Latinoamérica (Barrón et al., 2018; Papadópulos y Radakovich, 2006). 

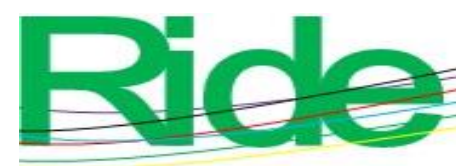

Revista Iberoamericana para la Investigación y el Desarrollo Educativo

ISSN $2007-7467$

Figura 1. Distribución por sexo y centro universitario

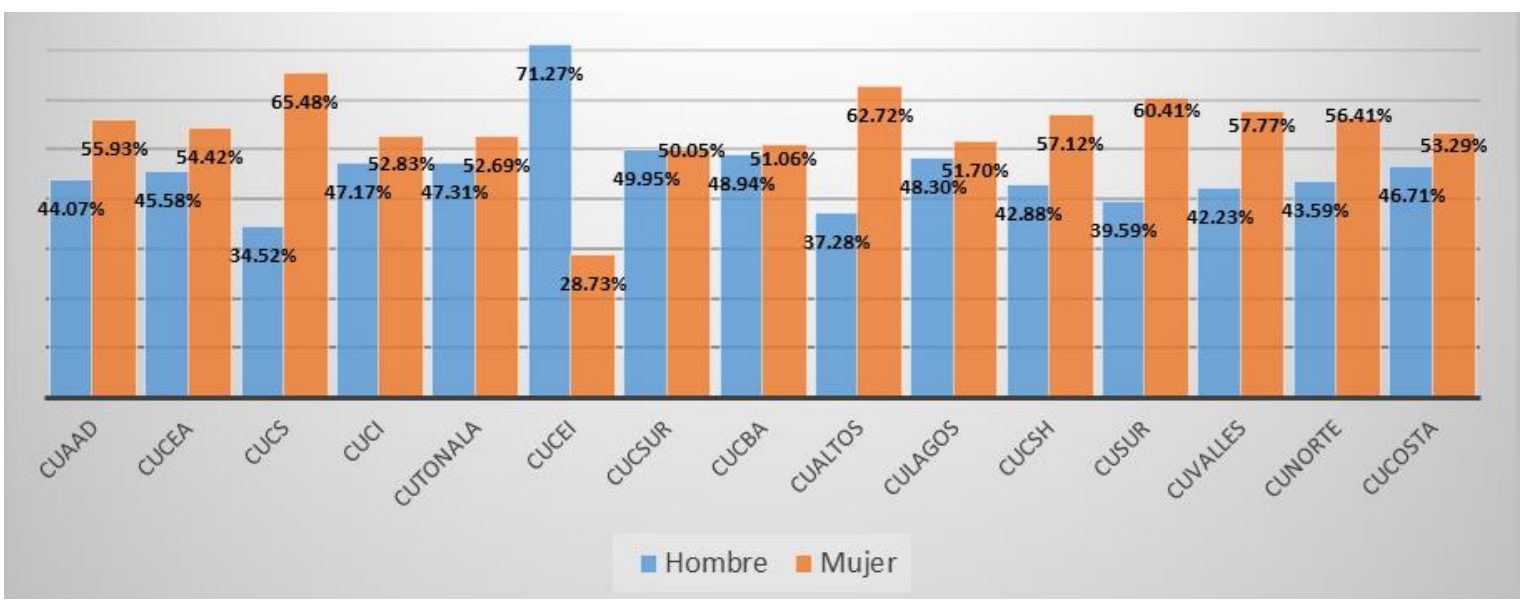

Fuente: Elaboración propia

Considerando todos los centros universitarios de la red, y respecto al estado civil de los estudiantes, se pueden apreciar diferencias significativas en los porcentajes por sexo (véase tabla 1). Se advierte un mayor porcentaje de mujeres casadas y divorciadas que hombres (2.35\% vs. $1.06 \%$ y $0.40 \%$ vs. $0.31 \%$, respectivamente), mientras que en los hombres es mayor la porción de solteros y viudos (95.95 \% vs. $94.65 \%$ y $0.18 \%$ vs. $0.10 \%$ ). El mismo patrón, pero más acentuado, se observa en los centros regionales para casadas y divorciadas ( $2.85 \%$ vs. $0.79 \%$ y 0.42 vs. $0.10 \%$, respectivamente) y para solteros y viudos (96.15\% vs. $94.47 \%$ y 0.30 vs. $0.08 \%$, respectivamente). Sin embargo, en los centros universitarios de la ZMG no existen diferencias significativas en los porcentajes por sexo (ver anexo, prueba 1). Una posible causa de que el porcentaje de mujeres casadas sea mayor que el de hombres se debe a que tradicionalmente (ya sea por razones culturales, por presión familiar o por embarazos no deseados) aquellas se casan en promedio más jóvenes que estos y este factor puede coincidir con la edad en la que cursan sus estudios universitarios. 


\section{Revista Iberoamericana para la Investigación y el Desarrollo Educativo ISSN 2007 - 7467}

Tabla 1. Estado civil por sexo

\begin{tabular}{|c|c|c|c|c|c|c|c|c|c|}
\hline & \multicolumn{3}{|c|}{ Todos } & \multicolumn{3}{|c|}{ Centros en la ZMG } & \multicolumn{3}{|c|}{ Centros regionales } \\
\hline Estado civil & 尺ి & 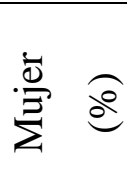 & 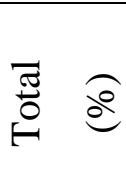 & 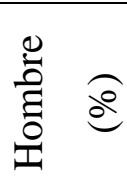 & $\cdot \stackrel{\vec{\Xi}}{\stackrel{\Xi}{\Xi}}$ & $\stackrel{\pi}{0}$ & 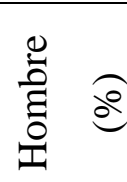 & $\stackrel{\dot{\bar{d}}}{\vec{z}} \widehat{\Theta}$ & $\stackrel{\pi}{0}$ \\
\hline Casado & 1.06 & 2.35 & 1.79 & 1.27 & 2.02 & 1.71 & 0.79 & 2.85 & 1.90 \\
\hline Divorciado & 0.31 & 0.40 & 0.36 & 0.48 & 0.39 & 0.43 & 0.10 & 0.42 & 0.27 \\
\hline Soltero & 95.95 & 94.65 & 95.21 & 95.78 & 94.77 & 95.19 & 96.15 & 94.47 & 95.24 \\
\hline Viudo & 0.18 & 0.10 & 0.13 & 0.08 & 0.11 & 0.10 & 0.30 & 0.08 & 0.18 \\
\hline Otro & 2.51 & 2.49 & 2.50 & 2.39 & 2.70 & 2.57 & 2.67 & 2.18 & 2.40 \\
\hline Total general & 100.0 & 100.0 & 100.0 & 100.0 & 100.0 & 100.0 & 100.0 & 100.0 & 100.0 \\
\hline & 0 & 0 & 0 & 0 & 0 & 0 & 0 & 0 & 0 \\
\hline
\end{tabular}

Fuente: Elaboración propia

Ahora bien, como puede apreciarse en la figura 2, los porcentajes de los estudiantes que son padres también presentan diferencias significativas: la proporción de mujeres que sí tienen hijos es considerablemente mayor a la de los hombres (4.57\% vs. $2.69 \%$ en todos los centros universitarios; $4.22 \%$ vs. $2.55 \%$ solo en los de la ZMG). Dicha tendencia es todavía más marcada en los centros regionales $(5.11 \%$ vs. $2.86 \%)$. Esto sugiere que en las zonas rurales las mujeres a nivel universitario son más propensas a embarazarse y tener hijos, en comparación con lo que sucede en las zonas urbanas (ver anexo, prueba 2).

Figura 2. Porcentaje de estudiantes que son padres por sexo

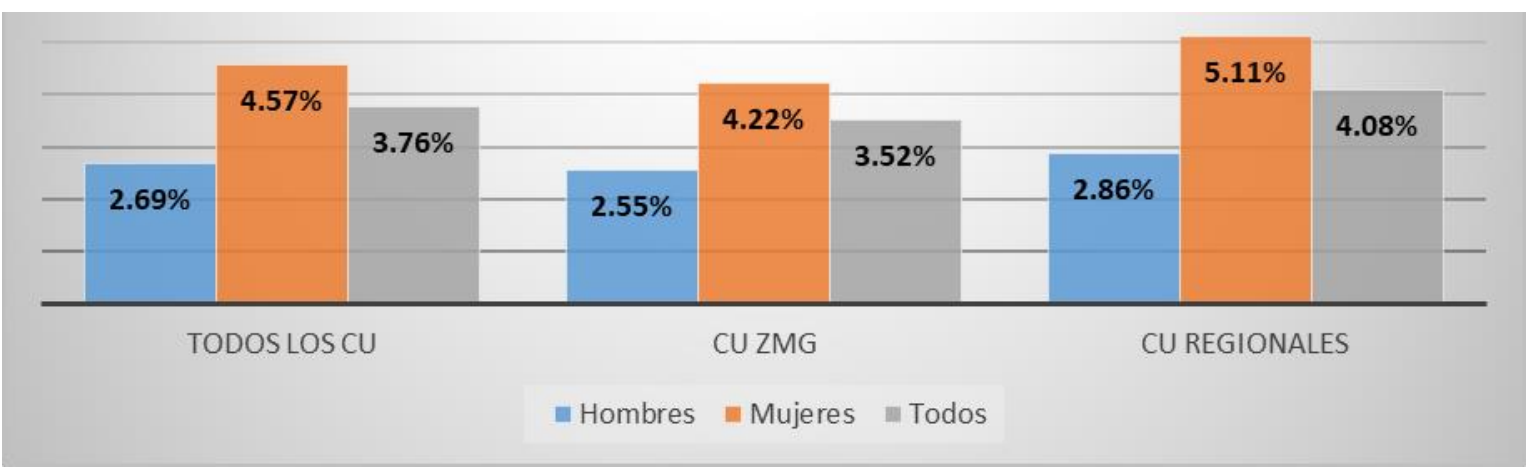

Fuente: Elaboración propia 


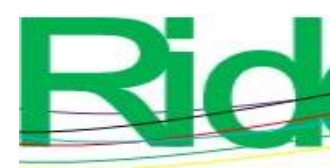

\section{Revista Iberoamericana para la Investigación y el Desarrollo Educativo ISSN 2007 - 7467}

Por lo que respecta al ingreso semanal promedio por sexo (ver figura 3$)^{1}$, si tomamos en cuenta todos los centros universitario, se nota que los hombres son más favorecidos que las mujeres, pues los niveles de ingresos más altos, de 500 a 1000 pesos y más 1000 pesos, son claramente superiores en los hombres (24.58\% y $18.30 \%$, respectivamente) en contraste con los mismos niveles de ingreso en las mujeres ( $12.33 \%$ y $5.58 \%$, respectivamente). Caso contrario se observa en los niveles más bajos de ingresos. Los niveles de menos de 150, de 150 a 300 y de 300 a 500 pesos presentan porcentajes claramente mayores en las mujeres $(12.18 \%, 34.75 \%$ y $29.20 \%$, respectivamente) que en los hombres (9.91\%, $26.34 \%$ y $26.83 \%$, respectivamente). En cuanto al nivel intermedio de 300 a 500 pesos, no se observan diferencias significativas. A nivel regional, esta tendencia es todavía más marcada que en la ZMG. Así pues, los resultados sugieren que existe, o bien cierta discriminación de los padres hacia las hijas, o bien los sueldos de los hombres que trabajan son mejor remunerados que los sueldos de las mujeres (véase anexo, prueba 3).

Figura 3. Porcentaje del ingreso semanal por sexo

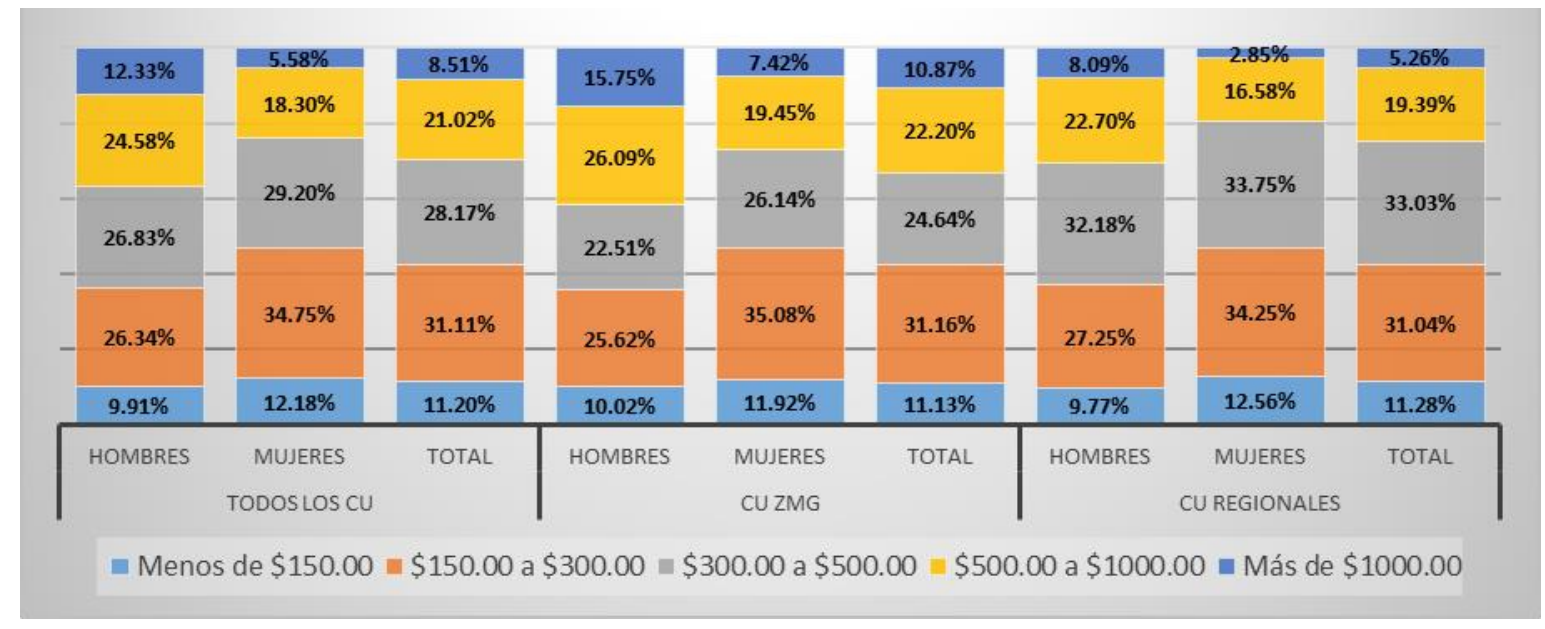

Fuente: Elaboración propia

\section{Indicadores académicos}

Según la UdeG (2019), tal y como puede apreciarse en la figura 4, las carreras con mayor porcentaje de hombres inscritos son ingeniería en Obras y Servicios (95.22\%), ingeniería Mecánica Eléctrica (94.42 \%), ingeniería Mecatrónica (93.49 \%), ingeniería en

${ }^{1}$ Este ingreso puede ser por remuneración laboral o por asignación familiar (generalmente a cargo de los padres). 

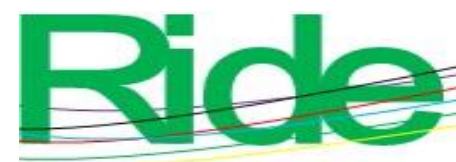

Revista Iberoamericana para la Investigación y el Desarrollo Educativo

ISSN $2007-7467$

Electrónica y Computación (92.31 \%) e ingeniería en Computación (89.86 \%). Mientras que las carreras con mayor porcentaje de mujeres son licenciatura en Trabajo Social (93.29 \%), licenciatura en Diseño de Interiores y Ambientación (89.66 \%), licenciatura en Enfermería $(85.71 \%)$, licenciatura en Nutrición (84.49 \%) y licenciatura en Diseño de Modas (84.37 \%). Es muy notorio que las carreras de ingeniería siguen siendo predominantemente masculinas, mientras que en las ramas de trabajo social, diseño y enfermería prevalece el sexo femenino, lo cual está en concordancia con el comportamiento a nivel nacional en la mayoría de las universidades (Huerta, 2017).

Figura 4. Carreras con mayor porcentaje de hombres y mujeres

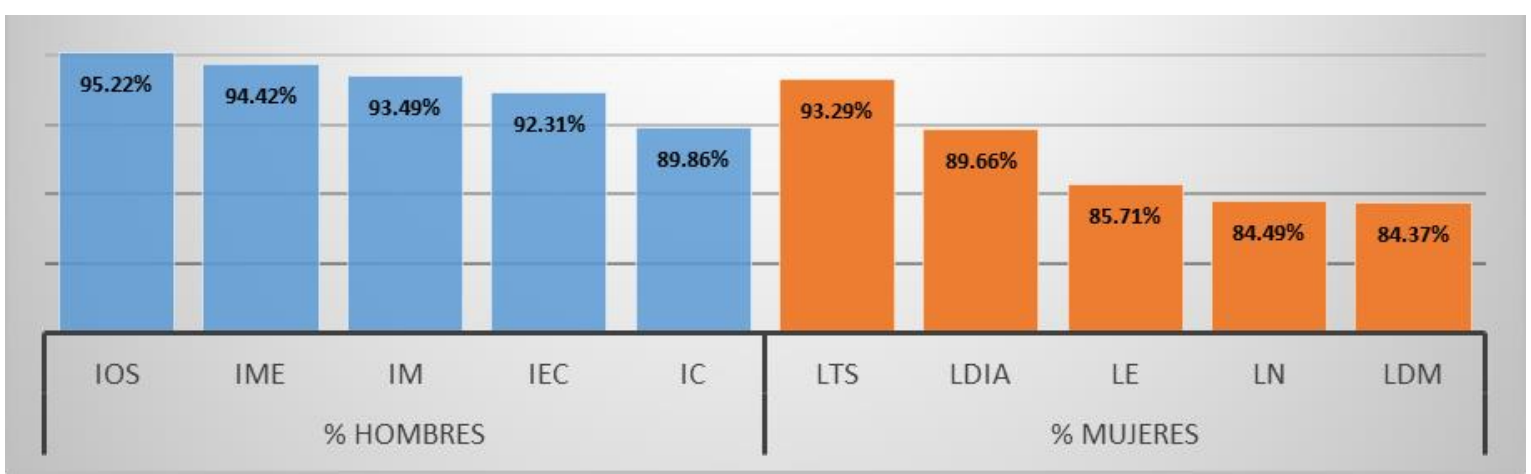

Fuente: Elaboración propia

Respecto a las calificaciones promedio por sexo, aquí sí se observan diferencias significativas. En general, el promedio es mayor en las mujeres. Si se toman en cuenta todos los centros universitarios, tenemos $84.75 \%$ en hombres vs. $86.40 \%$ en mujeres; al considerar solo los centros de la ZMG, tenemos $84.92 \%$ en hombres vs. $86.27 \%$ en mujeres, y en los centros regionales se registró $84.54 \%$ en hombres vs. $86.59 \%$ en mujeres, tal y como puede apreciarse en la figura 5 (véase anexo, prueba 4). Estos resultados pueden deberse al mayor número de horas dedicadas al estudio de parte de las mujeres (lo cual coincide con la información proporcionada en la figura 7), a que el porcentaje de mujeres que trabajan a nivel universitario sea menor que el de hombres (lo cual proporciona horas adicionales para estudiar) o simplemente a sus mejores hábitos de estudio. 


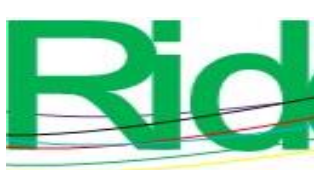

\section{Revista Iberoamericana para la Investigación y el Desarrollo Educativo ISSN 2007 - 7467}

Figura 5. Calificación promedio por sexo

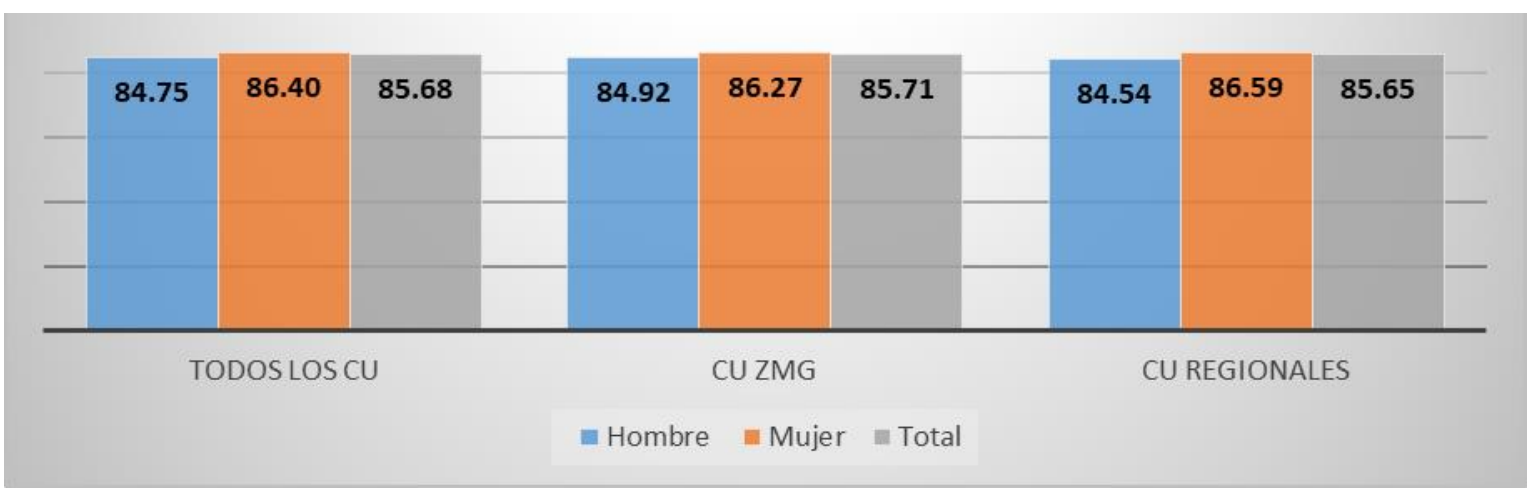

Fuente: Elaboración propia

En cuanto a la distribución por turno, si se toman en cuenta todos los centros universitarios o solo los de la ZMG (ver figura 6), no se advierten diferencias significativas en los porcentajes por sexo. Por el contrario, al observar exclusivamente el comportamiento de los centros regionales, el porcentaje de hombres en el turno matutino es significativamente mayor que el de las mujeres (45.10\% vs. $41.79 \%$ ) y que, de forma inversa, el porcentaje de mujeres en el turno vespertino es considerablemente mayor al porcentaje correspondiente de hombres $(29.48 \%$ vs. $24.33 \%)$. Esto puede deberse a que un mayor porcentaje de hombres trabaja por las tardes, mientras que las mujeres, en mayor proporción, se quedan en el hogar para ayudar con las labores domésticas (ver anexo, prueba 5).

Figura 6. Distribución del turno por sexo

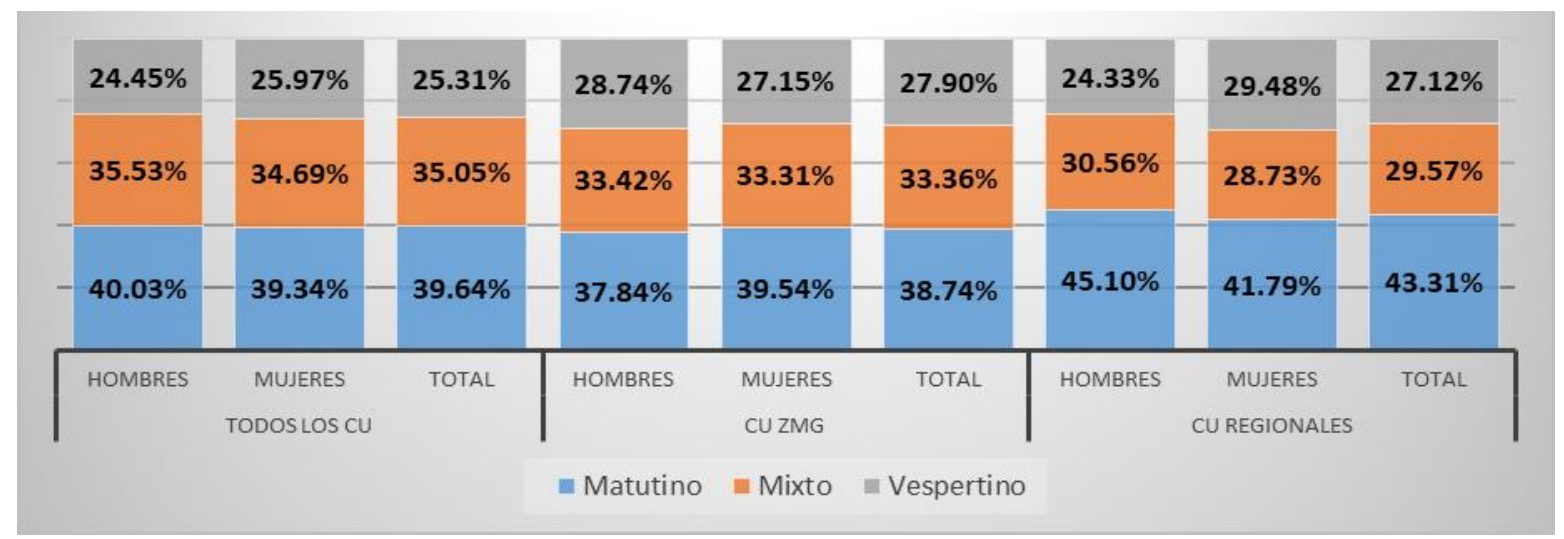

Fuente: Elaboración propia

Ahora bien, al analizar las horas que permanecen los alumnos en la escuela (ver figura 7), se notan diferencias significativas en los porcentajes por sexo. Así, los porcentajes correspondientes a los rangos de más horas de estadía diaria en la escuela (los rangos de seis 


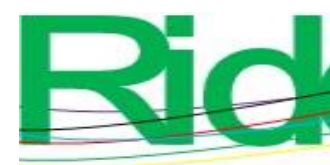

\section{Revista Iberoamericana para la Investigación y el Desarrollo Educativo ISSN 2007 - 7467}

a ocho horas y más de ocho horas) son claramente mayores en las mujeres que en los hombres (48.84 \% vs. $47.71 \%$ y $19.21 \%$ vs. $15.15 \%$, respectivamente); mientras que para los rangos de menos horas de permanencia en la escuela (de dos a cuatro horas y menos de dos horas) se cumple lo contrario ( $2.59 \%$ vs. $4.32 \%$ y $0.17 \%$ vs. $0.35 \%)$. Vale la pena advertir que dichos comportamientos son idénticos al considerar los centros de la ZMG y los regionales (ver anexo, prueba 6), lo cual sugiere que las mujeres son hasta cierto punto más dedicadas a las tareas académicas que los hombres; otra posible explicación es que tienen más tiempo libre y prefieren permanecer y desarrollar actividades extracurriculares en la escuela.

Figura 7. Permanencia diaria en la escuela por sexo

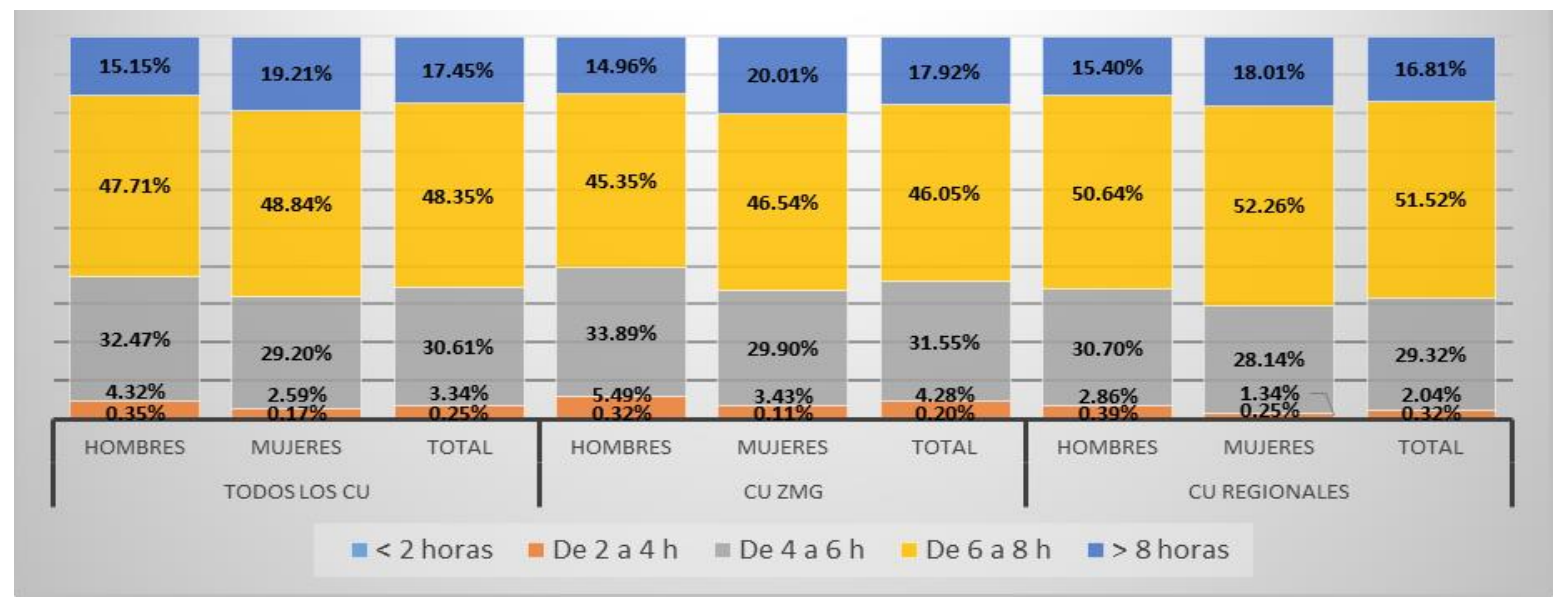

Fuente: Elaboración propia

\section{Orientación sexual}

En la figura 8 se pueden observar las orientaciones sexuales por sexo. Ahí se aprecia que el porcentaje de mujeres bisexuales es significativamente mayor al porcentaje correspondiente de hombres $(6.20 \%$ vs. $3.55 \%)$, mientras que el porcentaje de hombres homosexuales es significativamente mayor al porcentaje correspondiente de mujeres $(5.13 \%$ vs. $1.51 \%$ ). Por otro lado, los porcentajes de heterosexuales, tanto de hombres como de mujeres, no presentan diferencias significativas. También se notan algunas diferencias importantes entre los porcentajes de heterosexuales, por un lado, y homosexuales y bisexuales, por el otro. El porcentaje de heterosexuales en centros regionales es mayor al de los de la ZMG, tanto en hombres y mujeres como en el que suma a ambos sexos $(91.31 \%$, $92.29 \%$ y $91.84 \%$ vs. $89.10 \%, 90.39 \%$ y $89.86 \%$, respectivamente). Mientras que el 

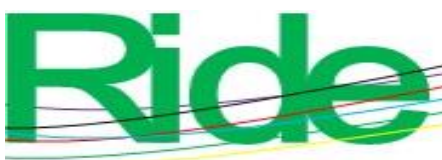

Revista Iberoamericana para la Investigación y el Desarrollo Educativo

ISSN $2007-7467$

porcentaje de homosexuales y bisexuales (la suma de ambos) en las sedes regionales es menor al mismo porcentaje en la ZMG; también aquí tanto en hombres y mujeres como el de ambos sexos $(8.68 \%, 7.71 \%$ y $8.15 \%$ vs. $10.90 \%, 9.61 \%$ y $10.14 \%$, respectivamente), lo que puede sugerir una mayor apertura en cuestiones sexuales en la población estudiantil de la ZMG que en la de las sedes regionales (véase anexo, prueba 7).

Figura 8. Orientación sexual por sexo

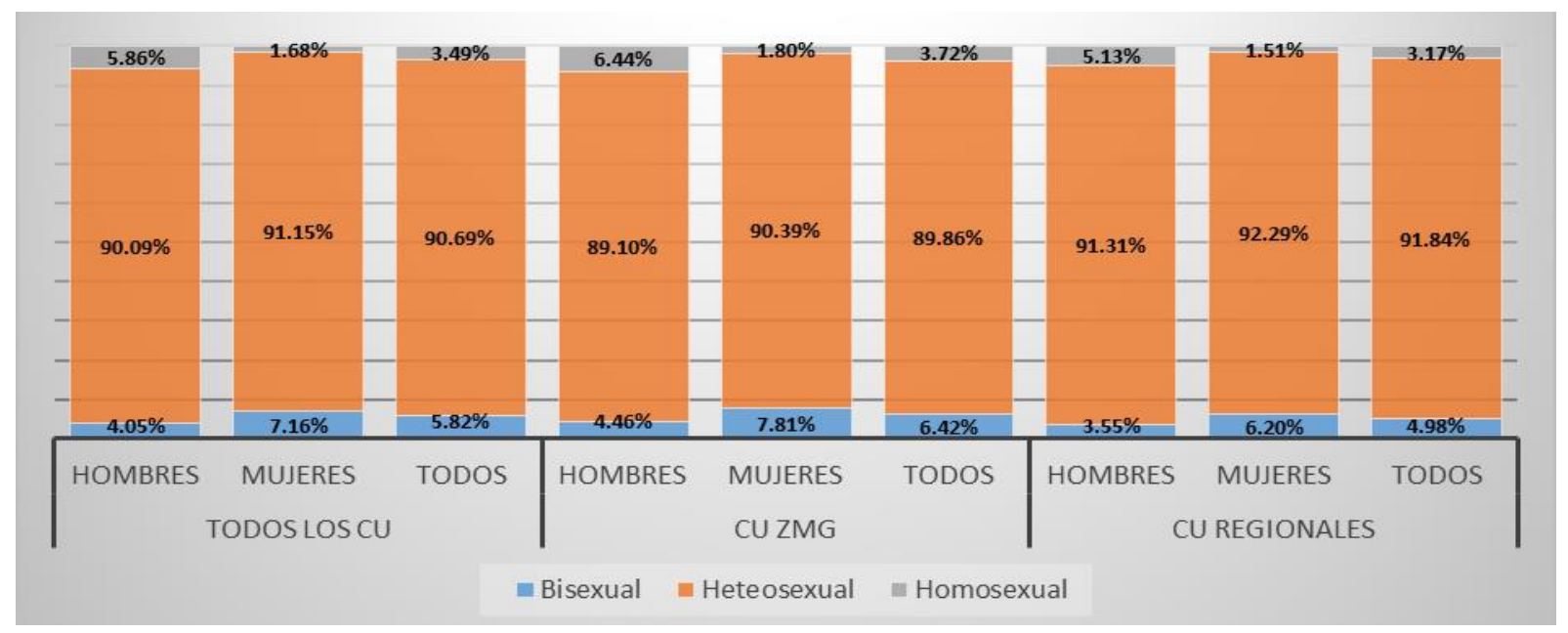

Fuente: Elaboración propia

En cuanto a quienes se identifican con la comunidad LGBTTTIQ, en los hombres predominan los gays y bisexuales (56\% y $36 \%$ respectivamente); mientras que en las mujeres prevalecen las bisexuales y las lesbianas (74 \% y $18 \%$ respectivamente) (ver figura 9). Cabe subrayar que entre esta y la figura anterior existe correspondencia, lo que corrobora el hecho de que, aun siendo categorías paralelas en hombres y mujeres, hay más gays que lesbianas. Esto sugiere que, si no existe algún factor biológico, psicológico o sociocultural que induzca esta condición, en los hombres parece ser más normal la aceptación de esta orientación sexual que en las mujeres. En ambos sexos también hay travestis, asexuales y quienes se identifican con la categoría queer, aunque en porcentajes mucho menores. 
Figura 9. Identificación con la comunidad LGBTTTIQ

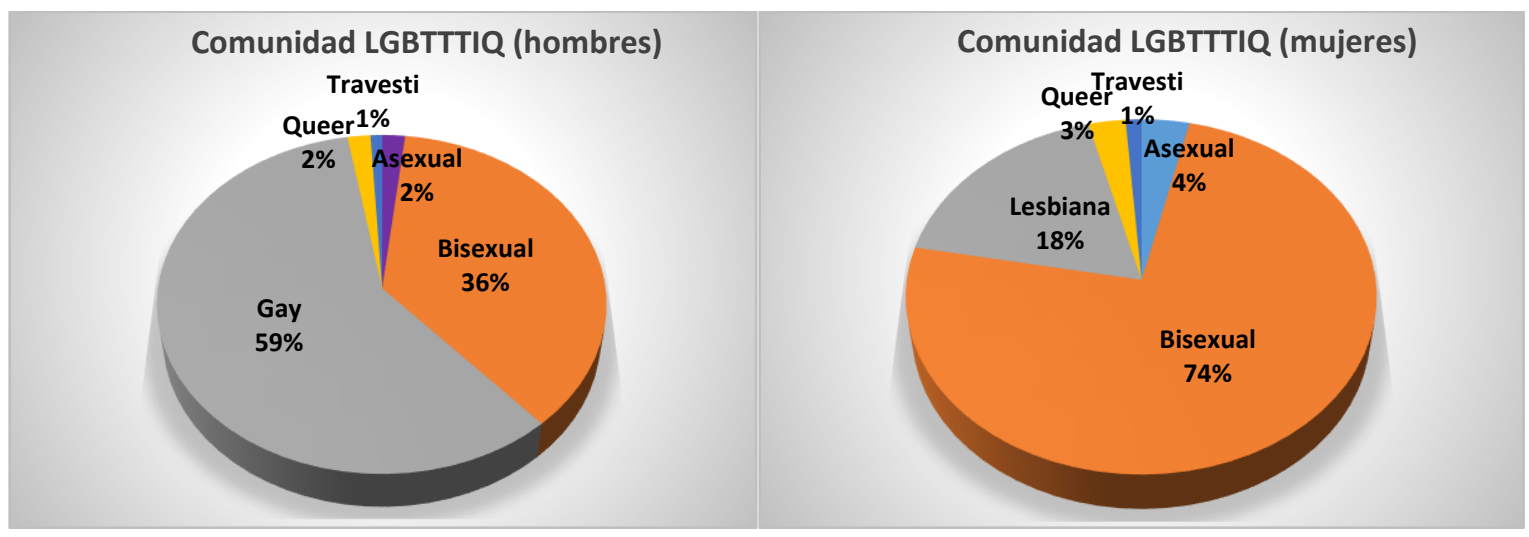

Fuente: Elaboración propia

\section{Adicciones}

En cuanto al tema de adicciones, se consultó sobre la ingesta de drogas, alcohol y tabaco. Por lo que respecta a las drogas (marihuana, cocaína, heroína, inhalantes, tranquilizantes, anfetaminas, extasis, LSD y similares), en los porcentajes por sexo de quienes han probado alguna vez drogas se pueden advertir diferencias significativas (ver figura 10). En todos los centros universitarios la proporción de hombres es claramente superior al de las mujeres (34.67\% vs. $22.57 \%$ ). Pero, si consideramos a los estudiantes de los centros universitarios de la ZMG, este comportamiento se acentúa más (40.73\% en hombres vs. $26.48 \%$ en mujeres). En los centros regionales, aunque se conserva este patrón (27.15\% en hombres vs. $16.75 \%$ en mujeres), los porcentajes son comparativamente menores. Lo anterior se puede deber a una conciencia más relajada con respecto al consumo de drogas en las zonas urbanas o por una mayor circulación de estupefacientes en la ciudad (véase anexo, prueba 8). 


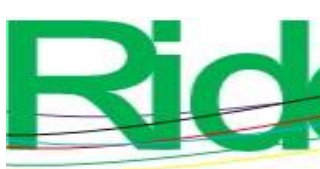

Revista Iberoamericana para la Investigación y el Desarrollo Educativo

ISSN $2007-7467$

Figura 10. Consumo de drogas por sexo

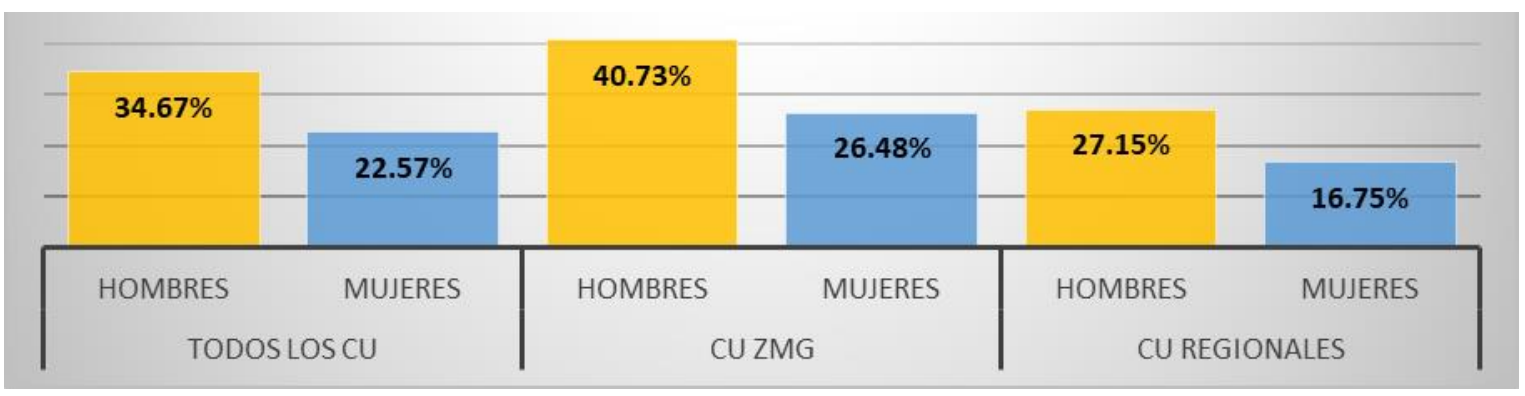

Fuente: Elaboración propia

A manera de complemento del gráfico anterior, en la figura 11 se puede observar que tal diferencia en el consumo de drogas puede ser explicada por la percepción de peligro que estas generan en uno y otro sexo (véase anexo, prueba 9). La escala "En extremo peligroso" es significativamente mayor en mujeres que en hombres tanto en todos los centros universitarios (30.99 \% vs. $24.50 \%$ ) como solo en los regionales (35.96 \% vs. $28.92 \%$ ) y solo en los centros de la ZMG (27.66 \% vs. $20.65 \%)$. Como es sabido, la percepción y el asumir riesgos es una característica de la configuración de ciertas masculinidades. En este sentido, el consumo puede ser incentivado por el grupo de amigos, por la presión que se ejerce y por el sentido de pertenencia que genera. Este comentario se puede extender también al alcohol y al tabaco.

Figura 11. Percepción del peligro por consumir drogas

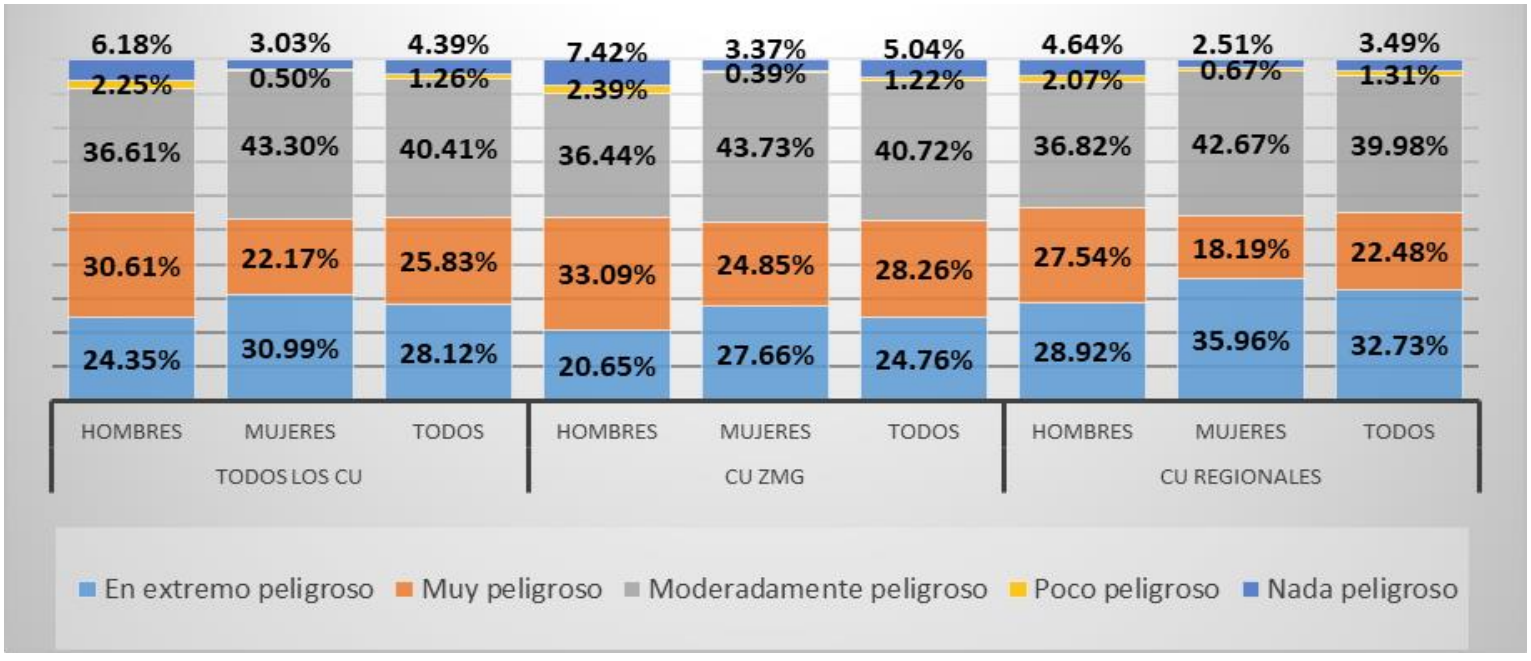

Fuente: Elaboración propia 


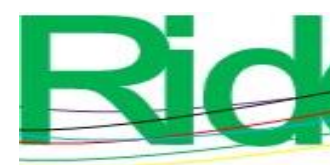

Revista Iberoamericana para la Investigación y el Desarrollo Educativo

ISSN 2007 - 7467

En lo que se refiere a la proporción de quienes han consumido alcohol alguna vez (ver figura 12), es significativamente mayor en hombres que en mujeres $(61.01 \%$ vs. $53.62 \%$, si consideramos todos los centros universitarios; $62.60 \%$ vs. $56.94 \%$ tomando en cuenta sólo los de la ZMG, y $56.56 \%$ vs. $48.66 \%$ para los regionales). Por otro lado, el consumo de alcohol se registra más alto en los centros de la ZMG que en los regionales tanto para hombres (64.60 \% vs. $56.56 \%$ ) como para mujeres (56.94 \% vs. $48.66 \%)$. Lo anterior muestra una mayor predisposición al consumo del alcohol en las zonas urbanas, que puede deberse al auge reciente de cantinas, centros botaneros y cervecerías en la ZMG (ver anexo, prueba 10).

Figura 12. Consumo de alcohol por sexo

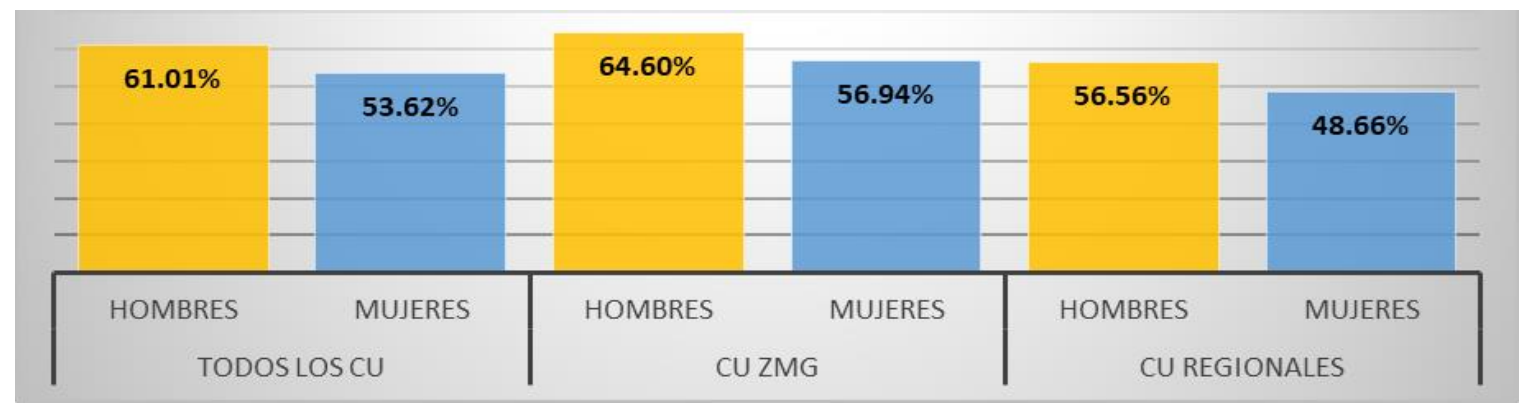

Fuente: Elaboración propia

Similarmente, en lo que se respecta a la proporción de quienes han consumido tabaco alguna vez (ver figura 13), el porcentaje correspondiente a hombres es mayor que el de las mujeres (20.22\% vs. $14.36 \%$ si consideramos todos los centros universitarios; $20.53 \%$ vs. $14.90 \%$ tomando en cuenta solo los de la ZMG, y $19.84 \%$ vs. $13.57 \%$ para los regionales). Al igual que con el alcohol, aunque más moderadamente, el consumo de tabaco es mayor para los centros universitarios de la ZMG en comparación con los regionales tanto para hombres (20.53 \% vs. $19.84 \%$ ) como para mujeres (14.90\% vs. $13.97 \%$ ). Y análogamente a lo que ocurre con el alcohol, esta mayor predisposición al consumo del tabaco en las zonas urbanas también puede deberse al auge de cantinas, centros botaneros y cervecerías en la ZMG (véase anexo, prueba 11). 


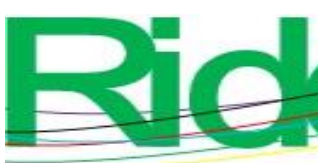

Revista Iberoamericana para la Investigación y el Desarrollo Educativo

ISSN $2007-7467$

Figura 13. Consumo de tabaco por sexo

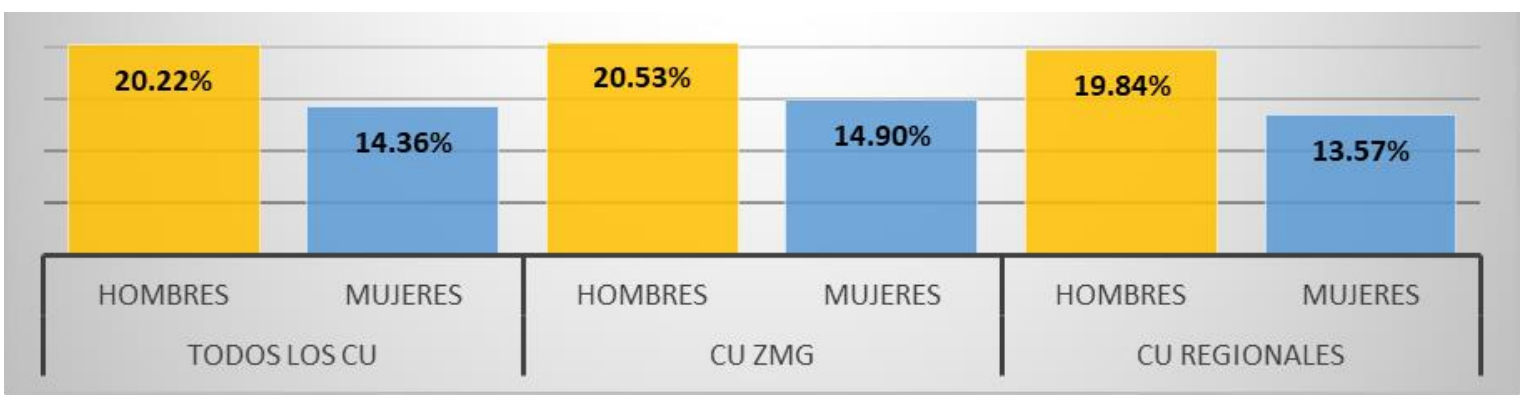

Fuente: Elaboración propia

\section{Bienestar}

Los niveles de felicidad y de los distintos tipos de satisfacción mostrados a continuación se tomaron de los estándares de la Comisión sobre la Medición del Desempeño Económico y el Progreso Social (también conocida como la Comisión Stiglitz-Sen-Fitoussi).

En cuanto a la percepción del nivel de felicidad de los estudiantes en su entorno (ver figura 14 y prueba 12 del anexo), los hombres son significativamente más felices, tanto en términos generales (78.64\% vs. $75.91 \%)$ como a nivel ZMG (78.02\% vs. $73.99 \%)$. Lo anterior puede explicarse, intuitivamente, por el hecho de que los hombres tienen mayor ingreso promedio, mayores oportunidades de trabajo y mayores libertades en el ambiente familiar, social y laboral. En los centros universitarios regionales no se aprecian diferencias significativas $(79.40 \%$ vs. $79.63 \%)$.

Figura 14. Percepción de la felicidad personal por sexo

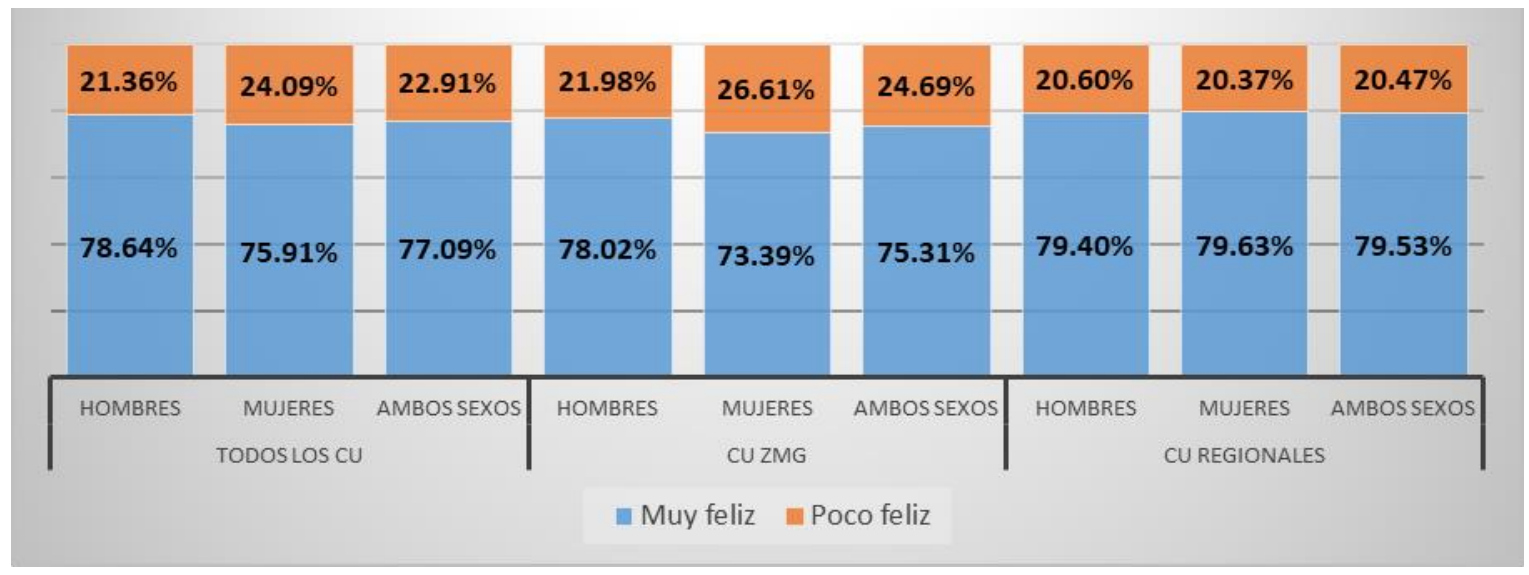

Fuente: Elaboración propia 


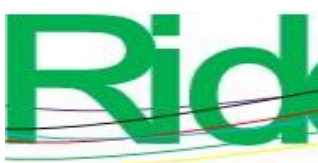

\section{Revista Iberoamericana para la Investigación y el Desarrollo Educativo ISSN 2007 - 7467}

Al analizar la satisfacción de los estudiantes respecto a su salud, se pueden observar diferencias importantes (ver figura 15 y prueba 13 del anexo). Por ejemplo, los hombres tienen un nivel de satisfacción mayor al de las mujeres. Los porcentajes en los niveles de muy satisfecho y satisfecho son claramente superiores $(31.43 \%$ vs. $23.05 \%$ en todos los centros, $28.71 \%$ vs. $19.37 \%$ en los de la ZMG y $34.80 \%$ vs. $28.51 \%$ para los regionales; $33.53 \%$ vs. $28.91 \%$ para todos los centros universitarios, $33.39 \%$ vs. $29.06 \%$ en los de la ZMG y $33.70 \%$ vs. $28.68 \%$ para los regionales, respectivamente). Y en los niveles de satisfacción inferiores el comportamiento sucede lo contrario: los porcentajes de los hombres son claramente inferiores. Así, en cuanto al nivel medio satisfecho, tenemos $22.25 \%$ vs. $28.40 \%, 24.84 \%$ vs. $30.88 \%$ y $19.04 \%$ vs. $24.70 \%$, general, ZMG y regional, en ese orden; en cuanto al nivel insatisfecho, $8.96 \%$ vs. $14.40 \%$ para todos los centros universitario, $9.27 \%$ vs. $15.61 \%$ en los de la ZMG y $8.57 \%$ vs. $12.61 \%$ para los regionales; y en cuanto al nivel muy insatisfecho, $3.83 \%$ vs. $5.24 \%$ para todos, $3.79 \%$ vs. $5.07 \%$ en los de la ZMG y $3.89 \%$ vs. $5.50 \%$ para los regionales. La razón por la cual los hombres se sienten más satisfacción de salud personal puede deberse a que las mujeres son culturalmente más cuidadosas con su salud, se sienten enfermas ante cualquier desequilibrio; por otro lado, los hombres suelen no quejarse y proseguir con sus actividades hasta que ya no puedan más o se den cuenta que tienen algún padecimiento o sufren alguna enfermedad, o tal vez transfieren la percepción de su salud a sus madres.

Figura 15. Satisfacción de la salud personal por sexo

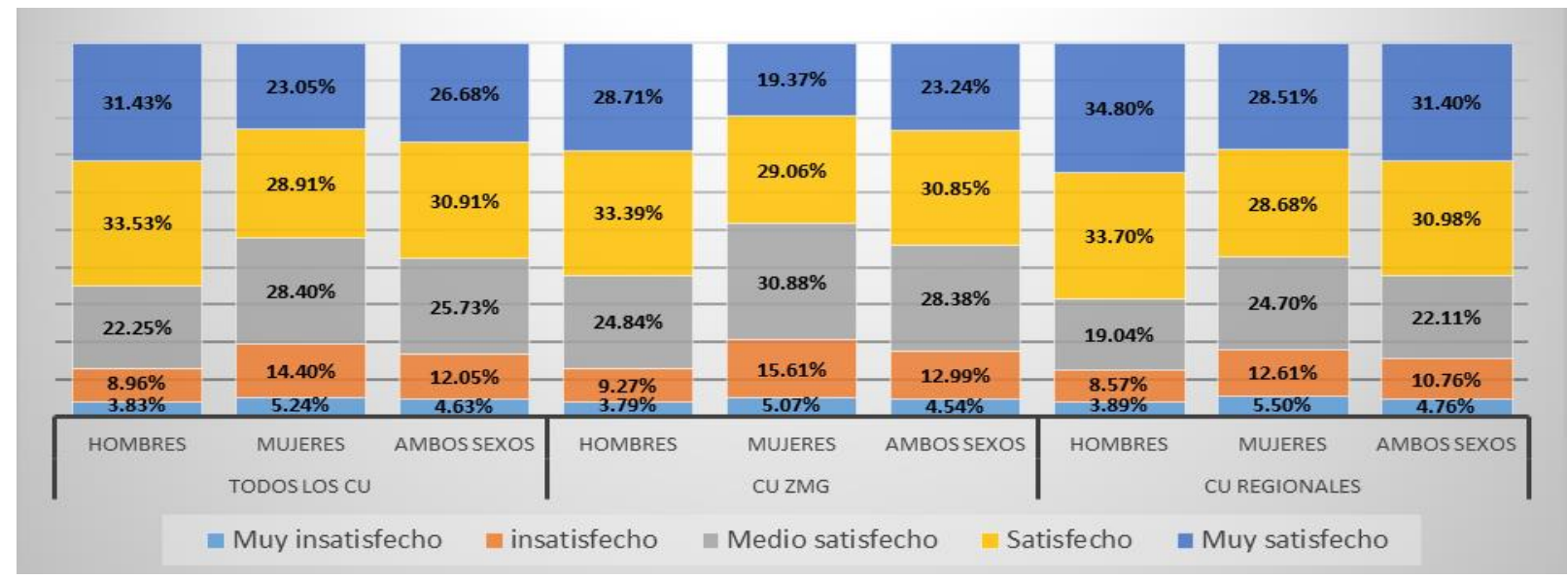

Fuente: Elaboración propia 


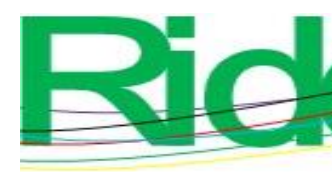

\section{Revista Iberoamericana para la Investigación y el Desarrollo Educativo ISSN 2007 - 7467}

En la figura 16, por su parte, se puede observar que los hombres tienen un nivel de satisfacción respecto a su economía personal mayor al de las mujeres: los porcentajes en los niveles de muy satisfecho y satisfecho son superiores (en el nivel muy satisfecho se registra $14.34 \%$ vs. $12.28 \%$ para todos los centros, $13.95 \%$ vs. $11.67 \%$ en los centros de la ZMG, y $14.83 \%$ vs. $13.19 \%$ para los centros universitarios regionales; y en el satisfecho $28.24 \%$ vs. $26.26 \%$ para todos, $27.59 \%$ vs. $25.50 \%$ en la ZMG y $29.05 \%$ vs. $27.39 \%$ a nivel regional). Mientras que para los niveles de satisfacción inferiores el comportamiento es contrario, y los porcentajes de los hombres son claramente inferiores; así, en el nivel medio satisfecho tenemos $31.93 \%$ vs. $32.93 \%$ para todos, $32.48 \%$ vs. $33.37 \%$ en la ZMG, y $31.24 \%$ vs. $32.27 \%$ en los regionales; en el nivel insatisfecho, $17.76 \%$ vs. $19.83 \%, 17.80 \%$ vs. $21.19 \%$ y $17.71 \%$ vs. $17.82 \%$; y en el nivel muy insatisfecho, $7.73 \%$ vs. $8.70 \%, 8.18 \%$ vs. $8.27 \%$ y $7.16 \%$ vs. $9.33 \%$, general, AMG y regional, respectivamente (véase anexo, prueba 14). Note que existe una concordancia clara entre el nivel de ingresos promedio (ver figura 4) y el nivel de satisfacción con respecto a la economía personal. En ambos casos, la situación de los hombres es más favorable.

Figura 16. Satisfacción de la economía personal por sexo

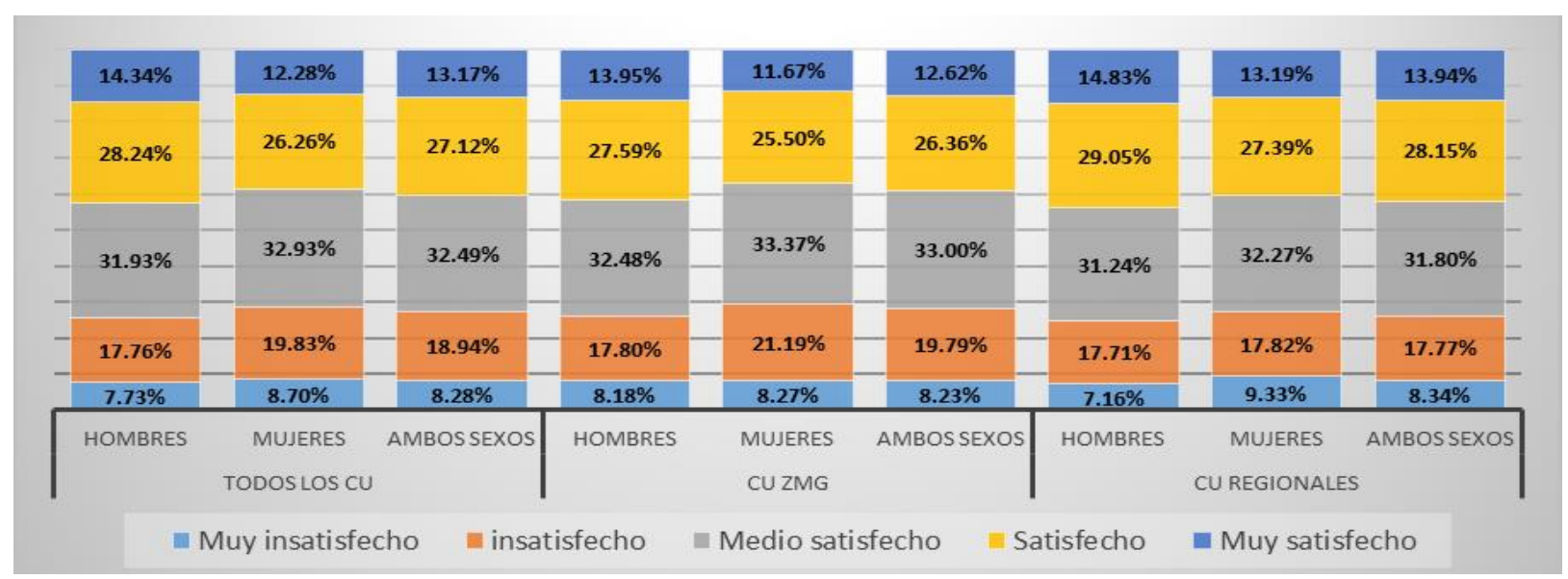

Fuente: Elaboración propia

Si analizamos la satisfacción de los estudiantes respecto a su ocupación, también se pueden advertir diferencias significativas (ver figura 17), de donde se deduce que los hombres tienen un nivel de satisfacción mayor al de las mujeres, para los niveles superiores; de esta forma en el nivel muy satisfecho tenemos, $18.74 \%$ vs. $16.54 \%$ para todos los CU, $18.08 \%$ vs. $14.71 \%$ en los CU de la ZMG, y $19.56 \%$ vs. $19.22 \%$ para los CU regionales; en cuanto al nivel satisfecho, $23.92 \%$ vs. $22.53 \%$ para todos los CU, $24.54 \%$ vs. $22.07 \%$ en los 
CU de la ZMG, y $23.15 \%$ vs. $22.27 \%$ para los CU regionales; y en cuanto al nivel medio satisfecho, $27.04 \%$ vs. $25.92 \%$ para todos los CU, $27.28 \%$ vs. $26.95 \%$ en los CU de la ZMG, y $26.75 \%$ vs. $24.39 \%$ para los CU regionales. Mientras que para el nivel más bajo, muy insatisfecho, tenemos que los porcentajes de los hombres son menores, $13.39 \%$ vs. $16.67 \%$ para todos los CU, $14.45 \%$ vs. $16.67 \%$ en los CU de la ZMG, y $12.08 \%$ vs. $16.68 \%$ para los CU regionales (ver prueba 15 del anexo). En este sentido, es claro, que aunque se han reducido las brechas por sexo en el mundo laboral, todavía existen diferencias notorias en salarios y oportunidades de trabajo entre hombres y mujeres, aun en países más desarrollados, por lo que la peor satisfacción ocupacional de las mujeres, sólo refleja tal situación (Aponte, 2008).

Figura 17. Satisfacción de la ocupación personal por sexo

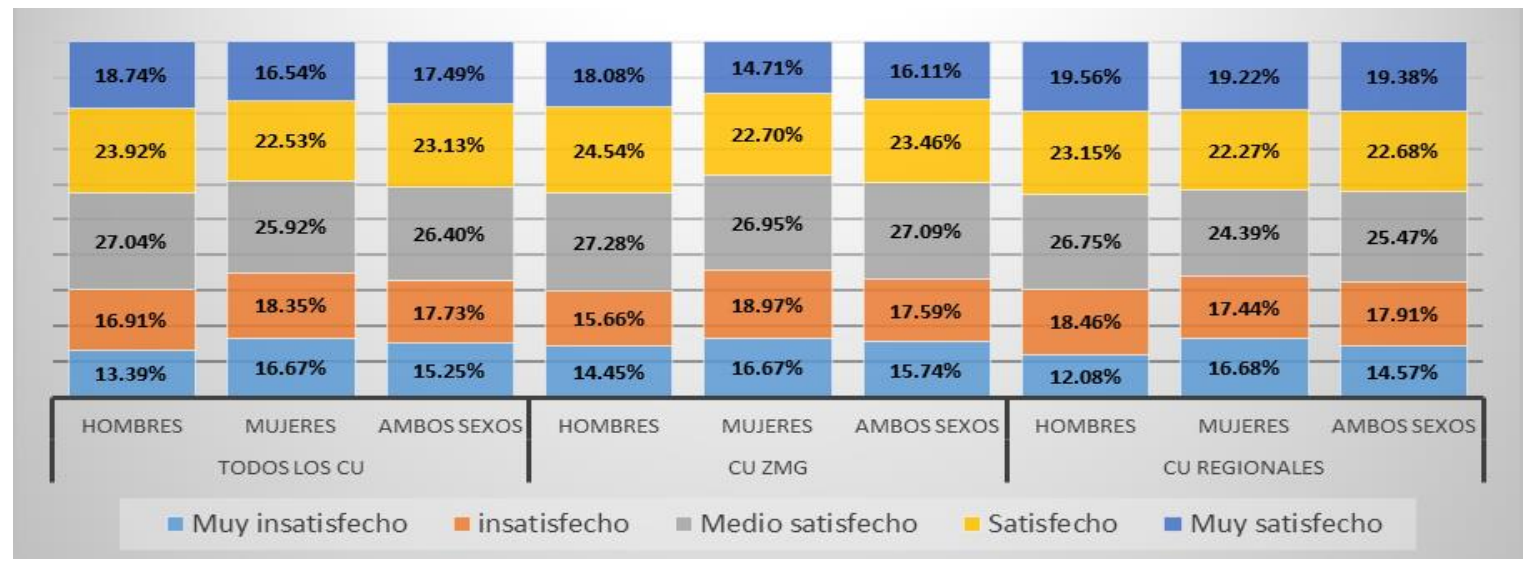

Fuente: Elaboración propia

Ahora bien, en la figura 18 se aprecia que los hombres tienen un nivel de satisfacción respecto a las relaciones familiares mayor al de las mujeres (véase anexo, prueba 16). Una posible explicación de esto es que en el entorno familiar tradicional se sigue valorando más el éxito en los hombres, pues, en el caso de que la mujer, aunque no sea exitosa académica y profesionalmente, puede conseguir, en última instancia, un buen marido. 


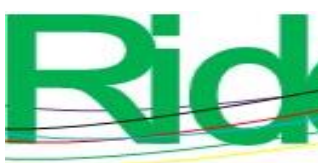

Figura 18. Satisfacción de las relaciones familiares por sexo

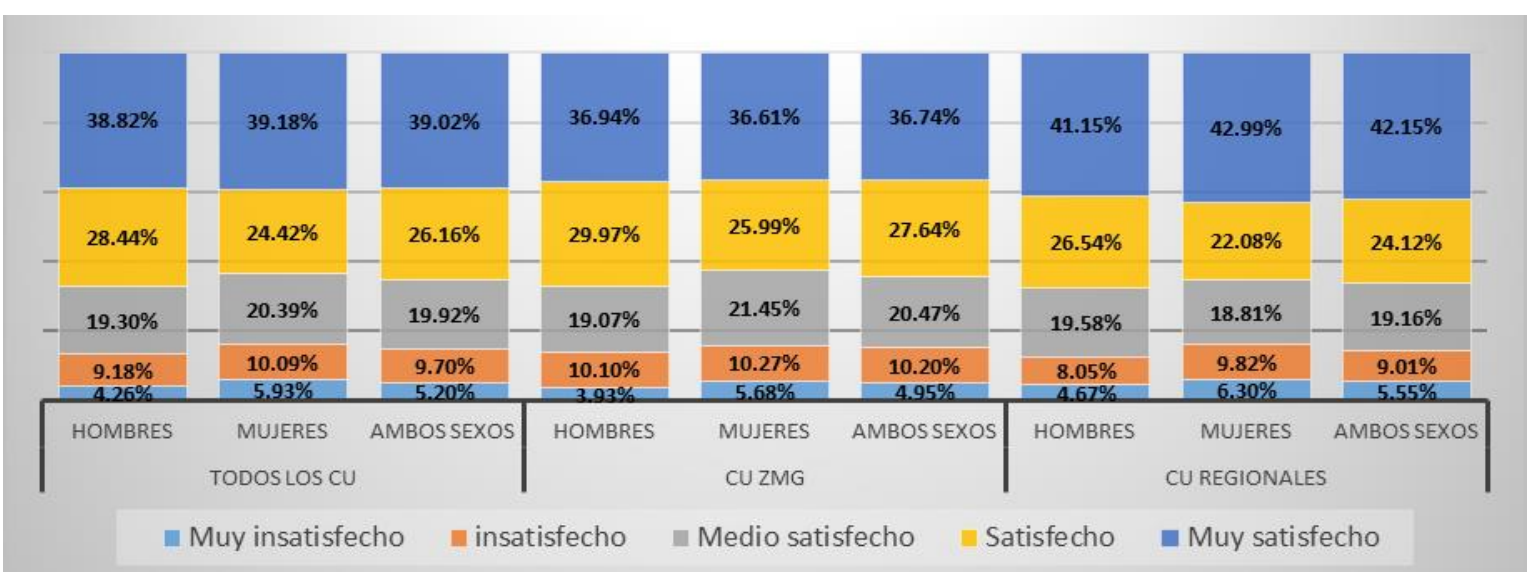

Fuente: Elaboración propia

En lo que se refiere a la satisfacción respecto a las relaciones amistosas, aunque los porcentajes por sexo también presentan diferencias significativas, no existe algún patrón de comportamiento claramente distinguible. Aun así, podemos mencionar algunas de las proporciones con mayor contraste (ver figura 19). Si consideramos todos los centros universitarios, en el nivel satisfecho tenemos $30.99 \%$ en hombres vs. $27.03 \%$ en mujeres; para los de la ZMG, en cuanto al nivel muy satisfecho, se tiene $50.46 \%$ en hombres vs. $45.55 \%$ en mujeres; y para los regionales, en cuanto al nivel muy satisfecho, se registra $39.44 \%$ en hombres vs. $43.93 \%$ en mujeres, y en cuanto al nivel satisfecho, $30.88 \%$ en hombres vs. $25.30 \%$ en mujeres (ver anexo, prueba 17). Los porcentajes anteriores muestran que la cuestión geográfica incide en la satisfacción de las relaciones amistosas por sexo. En los centros de la ZMG los hombres parecen tener relaciones afectivas más satisfactorias, mientras que en los centros universitarios regionales ocurre lo contrario, lo cual sugiere que la naturaleza las relaciones amistosas por sexo tiene un notorio ingrediente que refleja el contraste urbano-rural. 


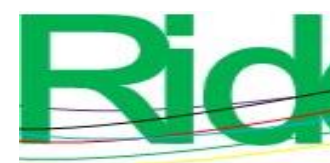

\section{Revista Iberoamericana para la Investigación y el Desarrollo Educativo ISSN 2007 - 7467}

Figura 19. Satisfacción en las relaciones amistosas por sexo

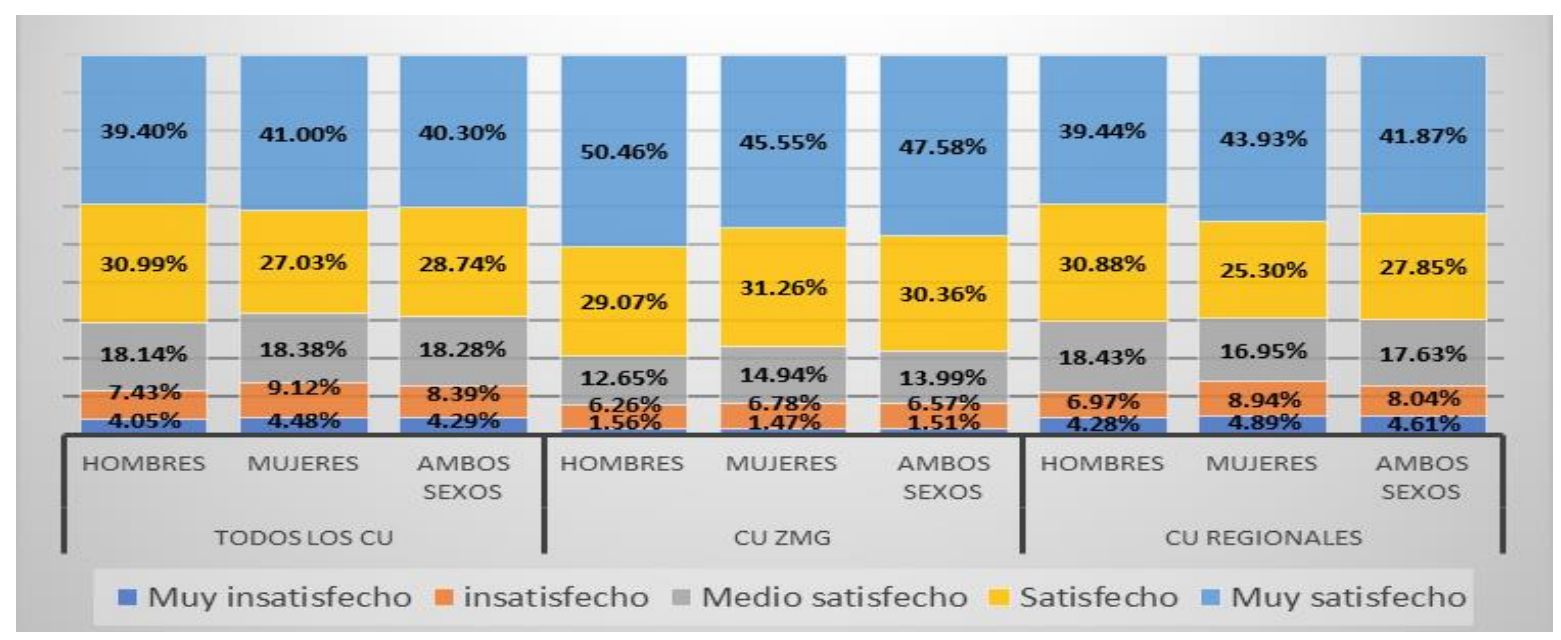

Fuente: Elaboración propia

Al estudiar la satisfacción de los estudiantes respecto al tiempo libre, también se pueden observar diferencias significativas (ver figura 20). Los hombres tienen un nivel de satisfacción mayor al de las mujeres (véase anexo, prueba 18). Los porcentajes en los niveles de muy satisfecho, satisfecho y medio satisfecho son superiores en los hombres. Mientras que para los niveles de satisfacción inferiores el comportamiento es inverso, y los porcentajes de los hombres son claramente inferiores. Una posible explicación de los resultados anteriores es que, tradicionalmente, el entorno familiar les proporciona mayor libertad de acción y de decisión a los hombres, mientras que a las mujeres se les limita y hasta cierto punto se les reprime.

Figura 20. Satisfacción en el tiempo libre por sexo

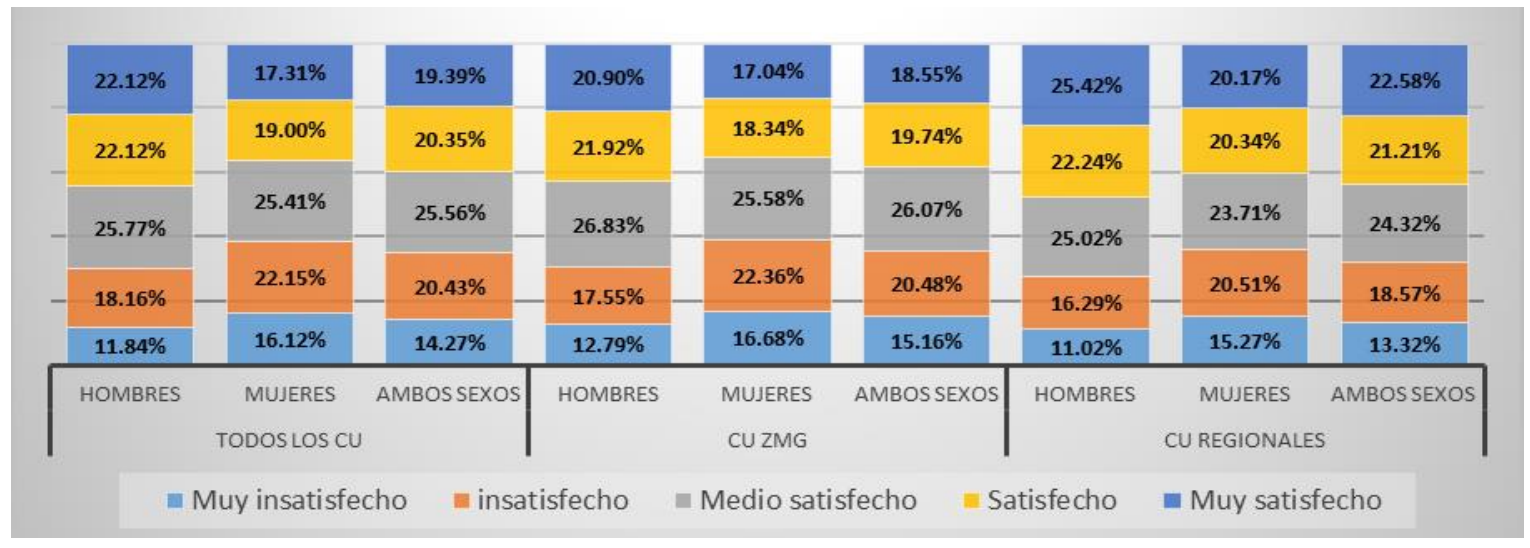

Fuente: Elaboración propia 


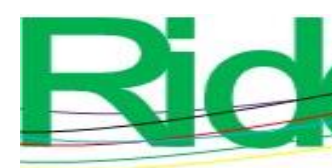

\section{Revista Iberoamericana para la Investigación y el Desarrollo Educativo ISSN 2007 - 7467}

Si consideramos la satisfacción de los estudiantes respecto al ambiente escolar, también se notan diferencias importantes (ver figura 21). Los hombres tienen un nivel de satisfacción mayor al de las mujeres; por ejemplo, los porcentajes en los niveles de muy satisfecho y satisfecho son claramente mayores (24.58\% vs. $20.08 \%$ para todos los centros, $23.89 \%$ vs. $17.83 \%$ en los de la ZMG y $25.42 \%$ vs. $23.43 \%$ para los regionales en cuanto al nivel muy satisfecho; y $34.46 \%$ vs. $32.89 \%$ para todos los centros universitarios, $33.79 \%$ vs. $33.05 \%$ en los de la ZMG y $35.29 \%$ vs. $32.66 \%$ para los regionales en cuanto al nivel satisfecho). Respecto a los bajos niveles de satisfacción, el comportamiento es inverso, y los porcentajes de los hombres son en la mayoría de los casos inferiores; así, en cuanto al nivel medio satisfecho tenemos $26.00 \%$ vs. $28.60 \%$ para todos los centros, $26.47 \%$ vs. $29.99 \%$ en los centros universitarios de la ZMG y $25.42 \%$ vs. $26.53 \%$ para los regionales; en cuanto al nivel insatisfecho, se registra $9.97 \%$ vs. $13.46 \%$ para todos los centros, $10.94 \%$ vs. $14.15 \%$ en los de la ZMG y $8.77 \%$ vs. $12.43 \%$ para los regionales; y en cuanto al nivel muy insatisfecho, por último, tenemos $4.99 \%$ vs. $4.97 \%$ para todos los centros universitarios, $4.91 \%$ vs. $4.98 \%$ en los de la ZMG y $5.08 \%$ vs. $4.95 \%$ para los centros universitarios regionales (ver anexo, prueba 19). Parece paradójico que las mujeres, aun teniendo mejor desempeño escolar, se sientan menos satisfechas, quizá porque su desempeño no se les reconoce en la misma medida y se sigue valorando más el de los hombres.

Figura 21. Satisfacción en el ambiente escolar por sexo

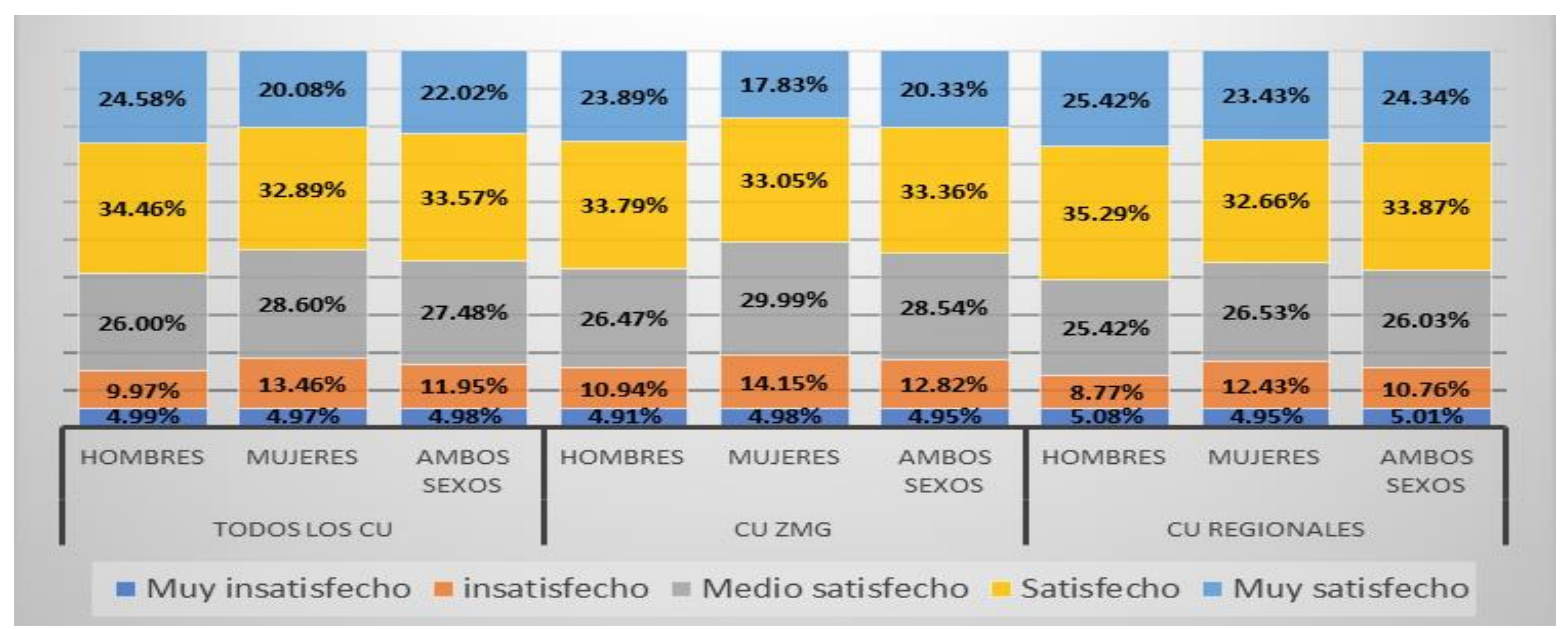

Fuente: Elaboración propia 


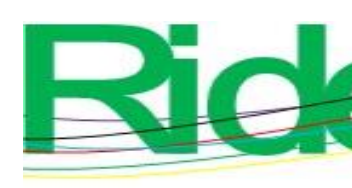

Revista Iberoamericana para la Investigación y el Desarrollo Educativo

ISSN $2007-7467$

A través del análisis gráfico, descriptivo e inferencial arriba presentado, que incluye muchas variables de interés, de distintos ámbitos, este trabajo ha demostrado su valía, pues revela el estado actual de las diferencias entre sexos que existen en la UdeG a nivel licenciatura. Aunado a ello, expone algunos motivos probables por los cuales se presentan estos resultados, que están sujetos a debate por la naturaleza general y exploratoria del trabajo. No obstante, la principal limitación de este trabajo radica en el carácter subjetivo de la encuesta, ya que los resultados dependen de la veracidad y honestidad con la que los estudiantes contestaron a las preguntas planteadas, es decir, las respuestas no se validaron y están supeditadas a la percepción de los estudiantes que respondieron la encuesta. Además, los autores no participaron en el diseño y aplicación de la encuesta; se tomó la información disponible de una base de datos pública. En trabajos futuros es recomendable focalizar las encuestas aplicadas a los estudiantes en ciertas esferas de interés, por ejemplo, en el rendimiento escolar, y validar en la medida de lo posible las respuestas recabadas de los alumnos, y así ganar en confianza y profundidad. A pesar de estas limitaciones, los resultados obtenidos pueden servir de utilidad para diseñar e implementar políticas educativas que tiendan a disminuir la brecha entre sexos en el entorno universitario y lograr un ambiente propicio en la vida universitaria, donde mujeres y hombres convivan con igualdad, respeto y armonía.

\section{Conclusiones}

El análisis estadístico llevado a cabo en este documento demuestra que todavía persisten marcadas diferencias entre sexos en diferentes ámbitos de la vida universitaria. También, gracias a la distinción entre centros universitarios regionales y centos de la ZMG, demuestra que existen algunas diferencias por sexo entre ambos contextos. En la mayoría de los rubros examinados se notan todavía marcados contrastes por sexo, algunos más acentuados que otros. Por ejemplo, en el ámbito académico, las mujeres mostraron un rendimiento promedio mayor; carreras con claras inclinaciones hacia uno y otro sexo fueron igualmente corroboradas. En cuestiones demográficas generales, el porcentaje de mujeres casadas y divorciadas es mayor, mientras que el porcentaje de hombres solteros y viudos es mayor; el porcentaje de madres es mayor al de padres entre los estudiantes; los hombres tienen un ingreso semanal mayor. En cuanto a la orientación sexual, el porcentaje de mujeres bisexuales es mayor al de hombres bisexuales, mientras que el porcentaje de hombres 

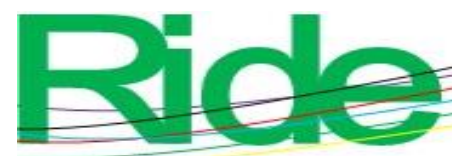

Revista Iberoamericana para la Investigación y el Desarrollo Educativo

ISSN 2007 - 7467

homosexuales es mayor al de mujeres homosexuales; entre los heterosexuales, los porcentajes no presentan diferencias significativas por sexo. Por otro lado, los hombres presentan una mayor propensión a las adicciones tanto si se consideran drogas, alcohol y tabaco. En la cuestión de bienestar, los hombres manifiestan ser más felices, así como tener mayor nivel de satisfacción en cuanto a su economía personal, su ocupación, sus relaciones familiares, su tiempo libre y sus relaciones en el ambiente escolar.

\section{Futuras líneas de investigación}

En el futuro se propone efectuar un exhaustivo estudio comparativo por sexo de rendimiento académico por área de conocimiento y por materia, aprovechando que se dispone de las calificaciones reales del alumnado por materia. El objetivo es identificar con precisión en qué disciplinas y áreas del saber existen diferencias significativas entre hombre y mujeres (y la magnitud de tales diferencias) y en cuáles el rendimiento es muy similar. La investigación propuesta es posible por centro universitario, ya que existe un núcleo importante de materias comunes a todas las carreras.

\section{Agradecimientos}

Los autores agradecen a la Federación de Estudiantes Universitarios por proporcionarnos la base de datos que sustenta los resultados de este estudio, y al doctor Juan Carlos Ramírez Rodríguez por sus valiosos comentarios al primer borrador de este trabajo. 


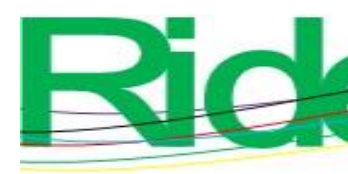

Revista Iberoamericana para la Investigación y el Desarrollo Educativo ISSN 2007 - 7467

\section{Referencias}

Abad, F., Ramírez, R., Fernandes, S. y Ramirez, R. (2019). Importancia del sexo/género y su distinción en la investigación biomédica. Hacia la Promoción de la Salud, 24(2), 1113. Recuperado de http://www.scielo.org.co/pdf/hpsal/v24n2/0121-7577-hpsal-2402-00011.pdf.

Aponte, E. (2008). Desigualdad, inclusión y equidad en la educación superior en América Latina y el Caribe: tendencias y escenario alternativo en el horizonte 2021. En Gazzola, A. L. y Didriksson, A. (eds.), Tendencias de la educación superior en América Latina y el Caribe (pp. 113-154). Caracas, Venezuela: Instituto Internacional de la Unesco para la Educación Superior en América Latina y el Caribe. Recuperado de http://beu.extension.unicen.edu.ar/xmlui/handle/123456789/303.

Barra, E. (2013). Bienestar psicológico y orientación de rol sexual en estudiantes universitarios. Terapia Psicológica, 28(1), 119-125. Recuperado de https://scielo.conicyt.cl/pdf/terpsicol/v28n1/art11.pdf.

Barrantes, K. y Ureña, P. (2015). Bienestar psicológico y bienestar subjetivo en estudiantes universitarios costarricenses. Revista Intercontinental de Psicología y Educación, 17(1), 101-123. Recuperado de https://www.redalyc.org/pdf/802/80242935006.pdf.

Barrón, K. S., Madera, J. A. y Cayeros, L. I. (2018). Mujeres universitarias y espacios de decisión: estudio comparativo en instituciones de educación superior mexicanas. Revista de La Educación Superior, 47(188), 39-56. Recuperado de http://www.scielo.org.mx/pdf/resu/v47n188/0185-2760-resu-47-188-39.pdf.

Del Valle, M., Hormaechea, F. y Urquijo, S. (2015). El bienestar psicológico: diferencias según sexo en estudiantes universitarios y diferencias con población general. Revista Argentina de Ciencias del Comportamiento, 7(3), 6-13. Recuperado de https://www.redalyc.org/pdf/3334/333443343003.pdf.

Fernández, T., Alguacil, J., Ayán, C., Bueno, A., Cancela, J. M., Capelo, R., Delgado, M., Jiménez, L., Jiménez, J. J., Llorca, J., Mateos, R., Molina, J., Velero, L. F. y Martín, V. (2013). Proyecto UNIHCOS cohorte dinámica de estudiantes universitarios para el estudio del consumo de drogas y otras adicciones. Revista Española de Salud Pública, 87(6), 575-585. Recuperado de http://scielo.isciii.es/pdf/resp/v87n6/03_colaboracion2.pdf. 


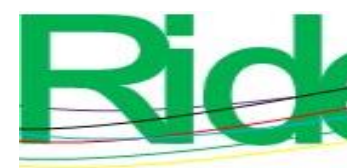

Revista Iberoamericana para la Investigación y el Desarrollo Educativo ISSN $2007-7467$

García, P. (2005). Género, educación y políticas públicas. La Ventana, 3(21), 70-89. Recuperado de http://www.scielo.org.mx/pdf/laven/v3n21/1405-9436-laven-3-2170.pdf.

Gómez, Z., Landeros, P., Noa, M. y Martínez, P. (2017). Consumo de alcohol, tabaco y otras drogas en jóvenes universitarios. Revista de Salud Pública y Nutrición, 16(4), 1-9. Recuperado de https://www.medigraphic.com/pdfs/revsalpubnut/spn2017/spn174a.pdf.

Huerta, R. M. (2017). Ingreso y presencia de las mujeres en la matrícula universitaria en México. Revista de El Colegio de San Luis, 7(14), 281-306. Recuperado de https://www.redalyc.org/pdf/4262/426252094011.pdf.

Lara, N., Saldaña, Y., Fernández, N. y Delgadillo, H. J. (2015). Salud, calidad de vida y entorno universitario en estudiantes mexicanos de una universidad pública. Hacia la Promoción de la Salud, 20(2), 102-117. Recuperado de http://www.scielo.org.co/pdf/hpsal/v20n2/v20n2a08.pdf.

Motos, P., Cortés, M. T., Giménez, J. A. y Cadaveira, F. (2015). Predictores del consumo semanal de alcohol y sus consecuencias asociadas en universitarios consumidores intensivos de alcohol. Adicciones, 27(2), 119-131. Recuperado de https://www.adicciones.es/index.php/adicciones/article/view/700/691.

Oswalt, S. B. and Wyatt, T. J. (2013). Sexual Health Behaviors and Sexual Orientation in a U.S. National Sample of College Students. Archives of Sexual Behavior, 42(8), 15611572. Retrieved from https://doi.org/10.1007/s10508-012-0066-9.

Oyarzún, G., Estrada, C., Pino, E. y Oyarzún, M. (2012). Habilidades sociales y rendimiento académico: una mirada desde el género. Acta Colombiana de Psicología, 15(2), 2128. Recuperado de https://dialnet.unirioja.es/servlet/articulo?codigo=5786536.

Pantoja, A. y Alcaide, M. (2013). La variable género y su relación con el autoconcepto y el rendimiento académico de alumnado universitario. Etic@net, 13(1), 124-140. Recuperado de http://eticanet.org/revista/index.php/eticanet/article/view/23/15.

Papadópulos, J. y Radakovich, R. (2006). Educación superior y género en América Latina y el Caribe. En Informe sobre la educación superior en América Latina y el Caribe 2000-2005. La metamorfosis de la educación superior (pp. 117-128). Caracas, Venezuela: Instituto Internacional de la Unesco para la Educación Superior en 


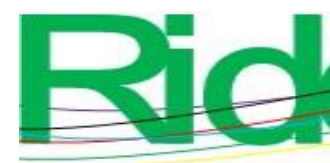

Revista Iberoamericana para la Investigación y el Desarrollo Educativo

ISSN 2007 - 7467

América Latina y el Caribe. Recuperado de https://www.ses.unam.mx/curso2013/pdf/Papadópulos 2006.pdf.

Paredes, E. y Polanski, T. (2016). Orientación sexual en una muestra de universitarios de Quito, Ecuador. Revista de Psicología Clínica con Niños y Adolescentes, 3(1), 19-24. Recuperado de http://www.dspace.uce.edu.ec/bitstream/25000/5326/1/Orientacion\%20sexual\%20e n\%20una\%20muestra.pdf.

Parker, S. W. y Pederzini, C. (2000). Género y educación en México. Estudios Demográficos y Urbanos, 15(1), 97-122. Recuperado de https://www.jstor.org/stable/40315023

Ramos, R., Duque, J. C. y Nieto, S. (2012). Un análisis de las diferencias rurales y urbanas en el rendimiento educativo de los estudiantes colombianos a partir de los microdatos de PISA. En Vieira, E. P. (coord. $\left.{ }^{a}\right)$, Investigaciones de economía de la educación (pp. 775-796). Asociación de Economía de la Educación. Recuperado de https://2012.economicsofeducation.com/user/pdfsesiones/177.pdf.

Torres, L. E. (2011). Rendimiento académico, familia y equidad de género. Ciencia y Sociedad, 36(1), 46-66. Recuperado de http://repositoriobiblioteca.intec.edu.do/handle/123456789/1352.

Universidad de Guadalajara [UdeG]. (2019). Informe de matrícula 2019A. Guadalajara, México: Universidad de Guadalajara. Recuperado de http://www.escolar.udg.mx/estadisticas/alumnos/informe-de-matricula-inicio-decurso-2019a. 


\begin{tabular}{|c|c|}
\hline Rol de Contribución & Autor (es) \\
\hline Conceptualización & Salvador Sandoval Bravo \\
\hline Metodología & $\begin{array}{l}\text { Salvador Sandoval Bravo, Víctor Hugo Gualajara Estrada } \\
\text { Grado de contribución: igual }\end{array}$ \\
\hline Software & No aplica \\
\hline Validación & $\begin{array}{l}\text { Salvador Sandoval Bravo, Víctor Hugo Gualajara Estrada, } \\
\text { Pedro Luis Celso Arellano } \\
\text { Grado de contribución: igual }\end{array}$ \\
\hline Análisis Formal & Salvador Sandoval Bravo \\
\hline Investigación & Salvador Sandoval Bravo \\
\hline Recursos & $\begin{array}{l}\text { Víctor Hugo Gualajara Estrada, Pedro Luis Celso Arellano } \\
\text { Grado de contribución: igual }\end{array}$ \\
\hline Curación de datos & Pedro Luis Celso Arellano \\
\hline $\begin{array}{l}\text { Escritura - Preparación del } \\
\text { borrador original }\end{array}$ & $\begin{array}{l}\text { Salvador Sandoval Bravo, Víctor Hugo Gualajara Estrada, } \\
\text { Pedro Luis Celso Arellano } \\
\text { Grado de contribución: igual }\end{array}$ \\
\hline $\begin{array}{l}\text { Escritura - Revisión y } \\
\text { edición }\end{array}$ & $\begin{array}{l}\text { Salvador Sandoval Bravo, Víctor Hugo Gualajara Estrada, } \\
\text { Pedro Luis Celso Arellano } \\
\text { Grado de contribución: igual }\end{array}$ \\
\hline Visualización & $\begin{array}{l}\text { Salvador Sandoval Bravo, Víctor Hugo Gualajara Estrada, } \\
\text { Pedro Luis Celso Arellano } \\
\text { Grado de contribución: igual }\end{array}$ \\
\hline Supervisión & $\begin{array}{l}\text { Víctor Hugo Gualajara Estrada, Pedro Luis Celso Arellano } \\
\text { Grado de contribución: igual }\end{array}$ \\
\hline Administración de Proyectos & $\begin{array}{l}\text { Víctor Hugo Gualajara Estrada, Pedro Luis Celso Arellano } \\
\text { Grado de contribución: igual }\end{array}$ \\
\hline Adquisición de fondos & No aplica \\
\hline
\end{tabular}




\section{Anexo}

Tabla 2. Pruebas e hipótesis respecto a si existen o no diferencias significativas por sexo en la muestra de estudio

\begin{tabular}{|c|c|c|c|c|}
\hline Prueba & Hipótesis & Todos & $\mathrm{ZMG}$ & Centros regionales \\
\hline 1 & $\begin{array}{l}\text { Ho: Los porcentajes por sexo } \\
\text { respecto al estado civil no } \\
\text { muestran diferencias } \\
\text { significativas. }\end{array}$ & $\begin{array}{l}\chi^{2}= \\
13.180 ; \chi_{\alpha}^{2}= \\
9.488 ; \alpha= \\
.05 . \text { Luego } \\
\text { rechazar Ho. }\end{array}$ & $\begin{array}{l}\chi^{2}= \\
\text { 2.983; } \chi_{\alpha}^{2}= \\
\text { 9.488; } \alpha= \\
\text {.05. Luego, no } \\
\text { rechazar Ho. }\end{array}$ & $\begin{array}{l}\chi^{2}=16.329 ; \chi_{\alpha}^{2}= \\
9.488 ; \alpha=.05 . \\
\text { Luego, rechazar Ho. }\end{array}$ \\
\hline 2 & $\begin{array}{l}\text { Ho: Los porcentajes por sexo } \\
\text { de quienes son padres no } \\
\text { muestran } \\
\text { significativas. }\end{array}$ & $\begin{array}{l}\chi^{2}= \\
12.678 ; \chi_{\alpha}^{2}= \\
3.841 ; \alpha= \\
.05 . \quad \text { Entonces, } \\
\text { rechazar Ho. }\end{array}$ & $\begin{array}{l}\chi^{2}= \\
6.042 ; \chi_{\alpha}^{2}= \\
3.841 ; \alpha= \\
.05 . \quad \text { Luego, } \\
\text { rechazar Ho. }\end{array}$ & $\begin{array}{l}\chi^{2}=7.068 ; \chi_{\alpha}^{2}= \\
3.841 ; \alpha=.05 . \\
\text { Luego, rechazar Ho. }\end{array}$ \\
\hline 3 & $\begin{array}{l}\text { Ho: Los porcentajes por sexo } \\
\text { respecto al ingreso promedio } \\
\text { semanal no muestran } \\
\text { diferencias significativas. }\end{array}$ & $\begin{array}{l}\chi^{2}= \\
130.817 ; \chi_{\alpha}^{2}= \\
7.815 ; \alpha= \\
.05 . \quad \text { Luego, } \\
\text { rechazar Ho. }\end{array}$ & $\begin{array}{l}\chi^{2}= \\
89.140 ; \chi_{\alpha}^{2}= \\
\text { 7.815; } \alpha= \\
.05 . \quad \text { Luego, } \\
\text { rechazar Ho. }\end{array}$ & $\begin{array}{l}\chi^{2}=52.164 ; \chi_{\alpha}^{2}= \\
7.815 ; \alpha=.05 . \\
\text { Luego, rechazar Ho. }\end{array}$ \\
\hline 4 & $\begin{array}{l}\text { Ho: Las calificaciones } \\
\text { promedio de las mujeres en } \\
\text { todos los CU es menor o } \\
\text { igual a la de los hombres. }\end{array}$ & $\begin{array}{l}t= \\
9.220 ; t_{\alpha}= \\
1.646 ; \alpha= \\
.05 . \text { Luego, } \\
\text { rechazar Ho. }\end{array}$ & $\begin{array}{l}t= \\
5.821 ; t_{\alpha}= \\
1.646 ; \alpha= \\
.05 . \text { Luego, } \\
\text { rechazar Ho. }\end{array}$ & $\begin{array}{l}t=7.289 ; t_{\alpha}= \\
1.646 ; \alpha=.05 \\
\text { Luego, rechazar Ho. }\end{array}$ \\
\hline 5 & $\begin{array}{l}\text { Ho: Los porcentajes por sexo } \\
\text { respecto al turno no muestran } \\
\text { diferencias significativas. }\end{array}$ & $\begin{array}{l}\chi^{2}= \\
1.581 ; \chi_{\alpha}^{2}= \\
5.991 ; \alpha= \\
.05 . \text { Luego, no } \\
\text { rechazar Ho. }\end{array}$ & $\begin{array}{l}\chi^{2}= \\
1.006 ; \chi_{\alpha}^{2}= \\
5.991 ; \alpha= \\
.05 . \text { Luego, no } \\
\text { rechazar Ho. }\end{array}$ & $\begin{array}{l}\chi^{2}=7.361 ; \chi_{\alpha}^{2}= \\
5.991 ; \alpha=.05 \\
\text { Luego, rechazar Ho. }\end{array}$ \\
\hline
\end{tabular}




\begin{tabular}{|c|c|c|c|c|}
\hline 6 & $\begin{array}{l}\text { Ho: Los porcentajes por sexo } \\
\text { respecto al tiempo que } \\
\text { permanecen en la escuela no } \\
\text { muestran diferencias } \\
\text { significativas. }\end{array}$ & $\begin{array}{l}\chi^{2}= \\
\text { 30.215; } \chi_{\alpha}^{2}= \\
9.488 ; \alpha= \\
.05 . \quad \text { Luego, } \\
\text { rechazar Ho. }\end{array}$ & $\begin{array}{l}\chi^{2}= \\
\text { 23.322; } \chi_{\alpha}^{2}= \\
\text { 9.488; } \alpha= \\
.05 . \quad \text { Luego, } \\
\text { rechazar Ho. }\end{array}$ & $\begin{array}{l}\chi^{2}=10.309 ; \chi_{\alpha}^{2}= \\
9.488 ; \alpha=.05 . \\
\text { Luego, rechazar Ho. }\end{array}$ \\
\hline 7 & $\begin{array}{l}\text { Ho: Los porcentajes por sexo } \\
\text { respecto a la orientación } \\
\text { sexual no muestran } \\
\text { diferencias significativas. }\end{array}$ & $\begin{array}{l}\chi^{2}= \\
62.017 ; \chi_{\alpha}^{2}= \\
5.991 ; \alpha= \\
.05 . \quad \text { Luego, } \\
\text { rechazar Ho. }\end{array}$ & $\begin{array}{l}\chi^{2}= \\
35.204 ; \chi_{\alpha}^{2}= \\
5.991 ; \alpha= \\
.05 . \quad \text { Luego, } \\
\text { rechazar Ho. }\end{array}$ & $\begin{array}{l}\chi^{2}=14.471 ; \chi_{\alpha}^{2}= \\
5.991 ; \alpha=.05 . \\
\text { Luego, rechazar Ho. }\end{array}$ \\
\hline 8 & $\begin{array}{l}\text { Ho: Los porcentajes por sexo } \\
\text { de quienes ya han consumido } \\
\text { drogas en todos los CU no } \\
\text { muestran diferencias } \\
\text { significativas. }\end{array}$ & $\begin{array}{l}\chi^{2}= \\
93.872 ; \chi_{\alpha}^{2}= \\
\text { 3.841; } \alpha= \\
.05 . \quad \text { Luego, } \\
\text { rechazar Ho. }\end{array}$ & $\begin{array}{l}\chi^{2}= \\
68.763 ; \chi_{\alpha}^{2}= \\
\text { 3.841; } \alpha= \\
.05 . \quad \text { Luego, } \\
\text { rechazar Ho. }\end{array}$ & $\begin{array}{l}\chi^{2}=35.072 ; \chi_{\alpha}^{2}= \\
3.841 ; \alpha=.05 . \\
\text { Luego, rechazar Ho. }\end{array}$ \\
\hline 9 & $\begin{array}{l}\text { Ho: Los porcentajes por sexo } \\
\text { respecto a la percepción del } \\
\text { consumo a las drogas no } \\
\text { muestran diferencias } \\
\text { significativas. }\end{array}$ & $\begin{array}{l}\chi^{2}= \\
129.986 ; \chi_{\alpha}^{2}= \\
9.488 ; \alpha= \\
.05 . \quad \text { Luego, } \\
\text { rechazar Ho. }\end{array}$ & $\begin{array}{l}\chi^{2}= \\
89.804 ; \chi_{\alpha}^{2}= \\
9.488 ; \alpha= \\
.05 . \quad \text { Luego, } \\
\text { rechazar Ho. }\end{array}$ & $\begin{array}{l}\chi^{2}=49.562 ; \chi_{\alpha}^{2}= \\
9.488 ; \alpha=.05 . \\
\text { Luego, rechazar Ho. }\end{array}$ \\
\hline 10 & $\begin{array}{l}\text { Ho: Los porcentajes por sexo } \\
\text { de quienes ya han consumido } \\
\text { alcohol no muestran } \\
\text { diferencias significativas }\end{array}$ & $\begin{array}{l}\chi^{2}= \\
28.708 ; \chi_{\alpha}^{2}= \\
3.841 ; \alpha= \\
.05 . \quad \text { Luego, } \\
\text { rechazar Ho. }\end{array}$ & $\begin{array}{l}\chi^{2}= \\
18.006 ; \chi_{\alpha}^{2}= \\
3.841 ; \alpha= \\
.05 . \quad \text { Luego, } \\
\text { rechazar Ho. }\end{array}$ & $\begin{array}{l}\chi^{2}=13.727 ; \chi_{\alpha}^{2}= \\
3.841 ; \alpha=.05 . \\
\text { Luego, rechazar Ho. }\end{array}$ \\
\hline 11 & $\begin{array}{l}\text { Ho: Los porcentajes por sexo } \\
\text { de quienes ya han consumido } \\
\text { tabaco no muestran } \\
\text { diferencias significativas. }\end{array}$ & $\begin{array}{l}\chi^{2}= \\
31.451 ; \chi_{\alpha}^{2}= \\
3.841 ; \alpha= \\
.05 . \quad \text { Luego, } \\
\text { rechazar Ho. }\end{array}$ & $\begin{array}{l}\chi^{2}= \\
16.368 ; \chi_{\alpha}^{2}= \\
3.841 ; \alpha= \\
.05 . \quad \text { Luego, } \\
\text { rechazar Ho. }\end{array}$ & $\begin{array}{l}\chi^{2}=15.699 ; \chi_{\alpha}^{2}= \\
3.841 ; \alpha=.05 . \\
\text { Luego, rechazar Ho. }\end{array}$ \\
\hline
\end{tabular}




\begin{tabular}{|c|c|c|c|c|}
\hline 12 & $\begin{array}{l}\text { Ho: Los porcentajes por sexo } \\
\text { de la percepción del nivel de } \\
\text { felicidad de los estudiantes } \\
\text { no muestran diferencias } \\
\text { significativas. }\end{array}$ & $\begin{array}{l}\chi^{2}= \\
5.374 ; \chi_{\alpha}^{2}= \\
3.841 ; \alpha= \\
.05 . \quad \text { Luego, } \\
\text { rechazar Ho. }\end{array}$ & $\begin{array}{l}\chi^{2}= \\
8.381 ; \chi_{\alpha}^{2}= \\
3.841 ; \alpha= \\
.05 . \quad \text { Luego, } \\
\text { rechazar Ho. }\end{array}$ & $\begin{array}{l}\chi^{2}=0.017 ; \chi_{\alpha}^{2}= \\
3.841 ; \alpha=.05 . \\
\text { Luego, no rechazar } \\
\text { Ho. }\end{array}$ \\
\hline 13 & $\begin{array}{l}\text { Ho: Los porcentajes por sexo } \\
\text { de la satisfacción de la salud } \\
\text { personal de los estudiantes no } \\
\text { muestran diferencias } \\
\text { significativas. }\end{array}$ & $\begin{array}{l}\chi^{2}= \\
97.654 ; \chi_{\alpha}^{2}= \\
9.488 ; \alpha= \\
.05 . \quad \text { Luego, } \\
\text { rechazar Ho. }\end{array}$ & $\begin{array}{l}\chi^{2}= \\
66.120 ; \chi_{\alpha}^{2}= \\
9.488 ; \alpha= \\
.05 . \quad \text { Luego, } \\
\text { rechazar Ho. }\end{array}$ & $\begin{array}{l}\chi^{2}=30.260 ; \chi_{\alpha}^{2}= \\
9.488 ; \alpha=.05 . \\
\text { Luego, rechazar Ho. }\end{array}$ \\
\hline 14 & $\begin{array}{l}\text { Ho: Los porcentajes por sexo } \\
\text { respecto a la satisfacción de } \\
\text { los estudiantes por } \quad \text { su } \\
\text { economía personal } \\
\text { muestran no } \\
\text { significativas }\end{array}$ & $\begin{array}{l}\chi^{2}= \\
\text { 10.697; } \chi_{\alpha}^{2}= \\
9.488 ; \alpha= \\
.05 . \quad \text { Luego, } \\
\text { rechazar Ho. }\end{array}$ & $\begin{array}{l}\chi^{2}= \\
8.649 ; \chi_{\alpha}^{2}= \\
9.488 ; \alpha= \\
.05 . \text { Luego, no } \\
\text { rechazar Ho. }\end{array}$ & $\begin{array}{l}\chi^{2}=4.817 ; \chi_{\alpha}^{2}= \\
9.488 ; \alpha=.05 . \\
\text { Luego, no rechazar } \\
\text { Ho. }\end{array}$ \\
\hline 15 & $\begin{array}{l}\text { Ho: Los porcentajes por sexo } \\
\text { respecto a la satisfacción de } \\
\text { los estudiantes en cuanto a su } \\
\text { ocupación personal no } \\
\text { muestran diferencias } \\
\text { significativas. }\end{array}$ & $\begin{array}{l}\chi^{2}= \\
15.656 ; \chi_{\alpha}^{2}= \\
9.488 ; \alpha= \\
.05 . \quad \text { Luego, } \\
\text { rechazar Ho. }\end{array}$ & $\begin{array}{l}\chi^{2}= \\
\text { 12.923; } \chi_{\alpha}^{2}= \\
9.488 ; \alpha= \\
.05 . \quad \text { Luego, } \\
\text { rechazar Ho. }\end{array}$ & $\begin{array}{l}\chi^{2}=9.611 ; \chi_{\alpha}^{2}= \\
9.488 ; \alpha=.05 \\
\text { Luego, rechazar Ho. }\end{array}$ \\
\hline 16 & $\begin{array}{l}\text { Ho: Los porcentajes por sexo } \\
\text { en relación a la satisfacción } \\
\text { de los estudiantes respecto a } \\
\text { sus relaciones familiares no } \\
\text { muestran diferencias } \\
\text { significativas. }\end{array}$ & $\begin{array}{l}\chi^{2}= \\
16.620 ; \chi_{\alpha}^{2}= \\
9.488 ; \alpha= \\
.05 . \quad \text { Luego, } \\
\text { rechazar Ho. }\end{array}$ & $\begin{array}{l}\chi^{2}= \\
\text { 10.761; } \chi_{\alpha}^{2}= \\
9.488 ; \alpha= \\
.05 . \quad \text { Luego, } \\
\text { rechazar Ho. }\end{array}$ & $\begin{array}{l}\chi^{2}=9.595 ; \chi_{\alpha}^{2}= \\
9.488 ; \alpha=.05 \\
\text { Luego, rechazar Ho. }\end{array}$ \\
\hline 17 & $\begin{array}{l}\text { Ho: Los porcentajes por sexo } \\
\text { en relación a la satisfacción } \\
\text { de los estudiantes respecto a }\end{array}$ & $\begin{array}{l}\chi^{2}= \\
12.758 ; \chi_{\alpha}^{2}= \\
9.488 ; \alpha=\end{array}$ & $\begin{array}{l}\chi^{2}= \\
4.892 ; \chi_{\alpha}^{2}= \\
9.488 ; \alpha=\end{array}$ & $\begin{array}{l}\chi^{2}=12.418 ; \chi_{\alpha}^{2}= \\
9.488 ; \alpha=.05 . \\
\text { Luego, rechazar Ho. }\end{array}$ \\
\hline
\end{tabular}




\begin{tabular}{|c|c|c|c|c|}
\hline & $\begin{array}{l}\text { sus amistades no muestran } \\
\text { diferencias significativas. }\end{array}$ & $\begin{array}{l}.05 . \quad \text { Luego, } \\
\text { rechazar Ho. }\end{array}$ & $\begin{array}{l}.05 . \text { Luego, no } \\
\text { rechazar Ho. }\end{array}$ & \\
\hline 18 & $\begin{array}{l}\text { Ho: Los porcentajes por sexo } \\
\text { en relación a la satisfacción } \\
\text { de los estudiantes respecto a } \\
\text { su tiempo libre no muestran } \\
\text { diferencias significativas. }\end{array}$ & $\begin{array}{l}\chi^{2}= \\
\text { 47.763; } \chi_{\alpha}^{2}= \\
\text { 9.488; } \alpha= \\
.05 . \quad \text { Luego, } \\
\text { rechazar Ho. }\end{array}$ & $\begin{array}{l}\chi^{2}= \\
28.419 ; \chi_{\alpha}^{2}= \\
9.488 ; \alpha= \\
.05 . \quad \text { Luego, } \\
\text { rechazar Ho. }\end{array}$ & $\begin{array}{l}\chi^{2}=20.580 ; \chi_{\alpha}^{2}= \\
9.488 ; \alpha=.05 . \\
\text { Luego, rechazar Ho. }\end{array}$ \\
\hline 19 & $\begin{array}{l}\text { Ho: Los porcentajes por sexo } \\
\text { en relación a la satisfacción } \\
\text { de los estudiantes respecto al } \\
\text { ambiente escolar no muestran } \\
\text { diferencias significativas. }\end{array}$ & $\begin{array}{l}\chi^{2}= \\
28.739 ; \chi_{\alpha}^{2}= \\
9.488 ; \alpha= \\
.05 . \quad \text { Luego, } \\
\text { rechazar Ho. }\end{array}$ & $\begin{array}{l}\chi^{2}= \\
22.369 ; \chi_{\alpha}^{2}= \\
9.488 ; \alpha= \\
.05 . \quad \text { Luego, } \\
\text { rechazar Ho. }\end{array}$ & $\begin{array}{l}\chi^{2}=9.037 ; \chi_{\alpha}^{2}= \\
9.488 ; \alpha=.05 . \\
\text { Luego, no rechazar } \\
\text { Ho. }\end{array}$ \\
\hline
\end{tabular}

Fuente: Elaboración propia 